\title{
BUILDING CARTESIAN PRODUCTS OF SURFACES WITH $[0,1]$
}

\author{
BY \\ ROBERT CRAGGS( $\left.{ }^{(}\right)$
}

1. Introduction. The following is a special case of Theorem 9.2, one of the chief results of this paper.

THEOREM. Suppose that $M$ is a 3-manifold with boundary, $S$ is a compact 2-manifold with boundary in $M$ such that $S \cap \mathrm{Bd}(M)=\mathrm{Bd}(S) \cap \mathrm{Bd}(M)=R$ either a 1-manifold with boundary or the empty set, and $\varepsilon>0$.

There is $a \delta>0$ such that if $f_{0}$ and $f_{1}$ are homeomorphisms of $S$ onto disjoint locally tame surfaces $S_{0}$ and $S_{1}$ in $M$ where $f_{e}(S) \cap \mathrm{Bd}(M)=f_{e}(R)$ and $f_{e}$ moves no point of $S$ by as much as $\delta(e=0,1)$, then there is a homeomorphism $g$ of $S \times[0,1]$ onto a locally tame solid in $M$ such that $g(S \times[0,1]) \cap \mathrm{Bd}(M)=g(R \times[0,1])$ and for each point $y$ of $S, g(y, e)=f_{e}(y)(e=0,1)$ and the diameter of $g(y \times[0,1])$ is less than $\varepsilon$.

This is the first of three papers (see also [13], [14]) where we investigate the global relation between two nice embeddings of a polyhedron in a 3-manifold with boundary where both embeddings approximate very closely a topological embedding of the polyhedron. In [13] we establish the following result.

THEOREM. Suppose that $M$ is a pwl 3-manifold (with boundary), $K$ is a finite polyhedron with no local cut points, $K_{a}$ is a subpolyhedron of $K$ with no degenerate components, $f$ is a homeomorphism of $K$ into $M$ such that $f(K) \cap \operatorname{Bd}(M)=f\left(K_{a}\right)$, and $\varepsilon>0$.

There is a $\delta>0$ such that if $f_{0}$ and $f_{1}$ are pwl homeomorphisms of $K$ into $M$ so that $f_{e}(K) \cap \operatorname{Bd}(M)=f_{e}\left(K_{a}\right)$ and $d\left(f, f_{e}\right)<\delta(e=0,1)$ where $d$ measures the distance between two maps of a space into a metric space, then there is a pwl $\varepsilon$-isotopy $H_{t}(0 \leqq t \leqq 1)$ of $M$ onto itself so that $H_{0}$ is the identity and $H_{1} f_{0}=f_{1}$.

In [14] we develop an analogous result involving regular neighborhoods for the case of embeddings of polyhedra which have local cut points.

Both theorems which we stated are obtained by refining techniques used in [5]. We hope that the serious reader will acquaint himself with that paper especially with the proof of Theorem 3.2 there. The reader should also be familiar with [12].

Received by the editors June 26, 1967.

(1) Some of the material here is taken from the author's Ph.D. thesis at the University of Wisconsin. We wish to thank Professor R. H. Bing for help in directing the thesis. Work here was supported by NSF grants GP 3857 and GP 5804. 
The lemmas of $\$ 2-6$ form a foundation on which we build the proofs of Theorem 7.1 here and Theorem 5.1 of [13]. In many cases the full strength of a lemma is needed only in [13].

In the remainder of this section we give several definitions. Many terms which we use are defined in [2]-[9], [12], and [25]. In particular our use of such terms as general position, normally situated, universal curve, $I(X, D)$, and Property $Q$ is explained in [12, $\$ 1$ and 6].

We use pwl as an abbreviation for piecewise linear(ly). A Euclidean complex is a rectilinear simplicial complex in some Euclidean space. A Euclidean polyhedron is a set which is the sum of the simplexes of a Euclidean complex. In general by complex we mean geometric simplicial complex. This is a partitioning of a separable metric space $X$ into subsets with affine structures by means of a homeomorphism $f:|K| \rightarrow X$ where $|K|$ denotes the polyhedron underlying a Euclidean complex $K$. The simplexes of a geometric complex are the images $f(s)$ of the simplexes $s$ of $K$. A polyhedron is the sum of the simplexes of a geometric complex. Suppose $X$ is a polyhedron which receives its pwl structure by way of a homeomorphism $f: P \rightarrow X$ where $P$ is a Euclidean polyhedron. We say a subset $Y$ of $X$ is a polyhedron in $\mathrm{X}$ or a subpolyhedron of $X$ if $f^{-1}(Y)$ is a Euclidean polyhedron. If $X$ and $Y$ are polyhedra receiving their pwl structures by homeomorphisms $f: P \rightarrow X$ and $g: Q \rightarrow Y$ where $P$ and $Q$ are Euclidean polyhedra then a map $h: X \rightarrow Y$ is pwl if $g^{-1} h f$ is a pwl map of $P$ into $Q$.

Suppose $X$ is a separable metric space. If $X$ is not regarded as a polyhedron then by a triangulation of $X$ we mean a geometric complex whose underlying point set is $X$. If $X$ is a polyhedron receiving its pwl structure by way of a homeomorphism $f: P \rightarrow X$ where $P$ is a Euclidean polyhedron, then a triangulation of $X$ is a geometric complex defined by means of a homeomorphism $g:|L| \rightarrow X$ such that $g^{-1} f$ is a pwl homeomorphism.

A pwl $n$-cell (n-sphere) is a polyhedron which is pwl homeomorphic to an $n$ simplex (the boundary of an $n+1$-simplex). A pwl $n$-manifold is a polyhedron such that each point has a closed polyhedral neighborhood which is a pwl $n$-cell. Notice that a pwl manifold can have a boundary.

Disjoint simplexes $r$ and $s$ in Euclidean space $E^{n}$ (or of a complex $L$ ) are joinable if there is a simplex $t$ of $E^{n}$ (resp. of $L$ ) whose vertices consist of the vertices of $r$ and $s$. We call $t$ the join of $r$ and $s$ and denote this join by $r s$. The empty simplex is allowed here and every simplex is joinable to it. Complexes $K_{1}$ and $K_{2}$ in $E^{n}$ (or subcomplexes $K_{1}$ and $K_{2}$ of a complex $L$ ) are joinable if each simplex of $K_{1}$ is joinable to each simplex of $K_{2}$ and the collection of joins of simplexes of $K_{1}$ with simplexes of $K_{2}$ together with their faces forms a complex (resp. a subcomplex of $L$ ). The complex just mentioned is called the join of $K_{1}$ and $K_{2}$ and is denoted by $K_{1} K_{2}$. Notice that local finiteness of complexes implies that only finite complexes can be joinable. Disjoint compact polyhedra $K_{1}$ and $K_{2}$ in $E^{n}$ are joinable if $K_{1}$ and $K_{2}$ possess rectilinear triangulations $T_{K_{1}}$ and $T_{K_{2}}$ which are joinable. In this 
case $K_{1} K_{2}$ denotes the join of $K_{1}$ and $K_{2}$ by which is meant the polyhedron that underlies $T_{K_{1}} T_{K_{2}}$.

If $K$ and $L$ are joinable polyhedra in $E^{m}$ and $f$ and $g$ are respectively maps of $K$ and $L$ into $E^{n}$, then by the join of the maps $f$ and $g$ we mean the map $h$ of $K L$ into $E^{n}$ which is given by $h(\alpha x+(1-\alpha) y)=\alpha f(x)+(1-\alpha) g(y)$ for $x \in K, y \in L$, and $0 \leqq \alpha \leqq 1$. We do not insist that $f$ and $g$ be pwl. If $K$ and $L$ are joinable polyhedra in $E^{m}$ and $f_{i} \rightarrow f$ and $g_{i} \rightarrow g$ are sequences of maps of $K$ and $L$ into $E^{n}$ which converge to maps $f$ and $g$, then the limit of the join of $f_{i}$ and $g_{i}$ is the join of $f$ and $g$.

If $s$ is a simplex in a complex $K$ then by $s t(s, K)$ we mean the polyhedron which is the sum of the simplexes of $K$ that have $s$ as a face. Since we take simplexes to be closed, st $(s, K)$ is compact. If $X$ is a set in a complex $K$ then by $N(X, K)$ we mean the polyhedron which is the sum of all simplexes of $K$ that intersect $X$.

A subcomplex $L$ of a complex $K$ is full in $K$ if for each simplex $s$ of $K, s$ belongs to $L$ if every proper face of $s$ belongs to $L$. A first derived subdivision $K^{\prime}$ of a complex $K$ is a subdivision of $K$ which is isomorphic to the first barycentric subdivision of $K$ under an isomorphism that takes each simplex of $K$ onto itself. An $n$th derived subdivision of a complex is defined by iteration. If $L$ is a subcomplex of a complex $K$, and $K^{\prime}$ is a first derived subdivision of $K$ with induced subdivision $L^{\prime}$ of $L$, then $L^{\prime}$ is full in $K^{\prime}$.

If $K$ is a finite polyhedron and $L$ is a subpolyhedron such that $K=L \cup B^{n}$ and $B^{n} \cap L=B^{n-1} \subset \mathrm{Bd}\left(B^{n}\right)$ where $B^{n}$ and $B^{n-1}$ are pwl $n$ - and $(n-1)$-cells, then we say that there is an elementary collapse from $K$ to $L$ or that $K$ collapses to $L(K \searrow L)$ by an elementary collapse. A polyhedron $K$ collapses to a subpolyhedron $L$ if there is a finite sequence of elementary collapses $K \searrow K_{1} \searrow K_{2} \cdots \searrow K_{n}=L$. We write this $K \searrow L$. If $T_{K}$ is a triangulation of $K$ in which each $\mathrm{Cl}\left(K_{i}-K_{i+1}\right)$ and each $\mathrm{Cl}\left(\mathrm{Bd}\left(\mathrm{Cl}\left(K_{i}-K_{i+1}\right)\right)-K_{i+1}\right)$ is a simplex of $T_{K}$, then $K$ collapses simplicially to $L$ (in $T_{K}$ ). A finite polyhedron is collapsible if it collapses to a point.

A finite polyhedron $N$ in a pwl $n$-manifold $M$ is a regular neighborhood of a finite polyhedron $K$ in $M$ if

1. $N$ is a pwl $n$-manifold,

2. $N$ contains a neighborhood of $K$ in $M$, and

3. $N$ collapses to $K$.

If a pwl manifold $M$ is a finite polyhedron which collapses to a subpolyhedron $K$, then $M$ is a (intrinsic) regular neighborhood of $K$. If $K$ is a finite polyhedron in a pwl manifold $M$, if $T$ is a triangulation of a neighborhood of $K$ in which $K$ underlies a full subcomplex, and if $T^{\prime}$ is a first derived subdivision of $T$, then we call $N\left(K, T^{\prime}\right)$ a derived neighborhood of $K$ in $M$.

In [24], [25] it is shown that if $K$ is a finite polyhedron in a pwl manifold $M$ then (1) a derived neighborhood of $K$ in $M$ is a regular neighborhood, (2) any two regular neighborhoods of $K$ in $M$ are pwl homeomorphic under a homeomorphism that is the identity on $K$, and (3) a regular neighborhood of $K$ is a pwl cell if $K$ is collapsible. 
We use $[a, b]$ to denote the closed interval $a \leqq t \leqq b$ and $(a, b)$ to denote the open interval $a<t<b$. Half open intervals are denoted by $[a, b)$ and $(a, b]$.

An isotopy $H_{t}(a \leqq t \leqq b)$ of a space $X$ into a space $Y$ is a continuous one parameter family of embeddings of $X$ into $Y$. An ambient isotopy $H_{t}(a \leqq t \leqq b)$ of a space $X$ or an isotopy of a space $X$ onto itself is a continuous family of homeomorphisms of $X$ onto itself. For such an isotopy we assume that $H_{a}$ is the identity if $a=0$. Associated with an isotopy $H_{t}(a \leqq t \leqq b)$ of a space $X$ into a space $Y$ is a level preserving homeomorphism $H: X \times[a, b] \rightarrow Y \times[a, b]$ which is given by the rule $H(x, t)=\left(H_{t}(x), t\right)$.

Two definitions of the term pwl isotopy appear in the literature. Bing and Sanderson [5], [22] call an isotopy $H_{t}(a \leqq t \leqq b)$ of a polyhedron $K$ into a polyhedron $L$ piecewise linear if for each value of $t, H_{t}$ is a pwl homeomorphism of $K$ into $L$. We prefer to follow Gugenheim [16] and say that $H_{t}$ is piecewise linear if the associated homeomorphism $H: K \times[a, b] \rightarrow L \times[a, b]$ is pwl. These two definitions are not equivalent, and this inequivalence causes us some difficulty since we employ isotopy results of [5], [22] involving isotopies that are not pwl in the Gugenheim sense. We get around this difficulty by showing how to approximate certain isotopies by pwl ones.

By the track of a set $Z$ under an isotopy $H_{t}(a \leqq t \leqq b)$ is meant $\bigcup\left\{H_{t}(Z) \mid t \in[a, b]\right\}$. An isotopy $H_{t}(a \leqq t \leqq b)$ of a space $X$ into a metric space $Y$ is an $\varepsilon$-isotopy if the track under $H_{t}$ of each point of $X$ has diameter less than $\varepsilon$.

A cylindrical sphere in $E^{3}$ with axis $L$ is the boundary of a 3-cell that is obtained by taking the closure of the part of a solid cylinder with axis $L$ that lies between two planes perpendicular to $L$. The bases of the cylindrical sphere are the two 2-cells which compose the intersection of the sphere with the two planes just mentioned.

We use $\rho$ to denote the metric on a metric space and $I$ to denote the identity homeomorphism. If $f$ and $g$ are maps of a space $X$ into a metric space $Y$ we denote by $d(f, g)$ the least upper bound over points $x$ of $X$ of the distances $\rho(f(x), g(x))$. We will often be occupied with extending homeomorphisms defined on subpolyhedra of polyhedra. In order to conserve on symbols we will usually denote an extension of a homeomorphism with the same symbol used to denote the homeomorphism.

Consider a finite collection $D_{1}, \ldots, D_{n}$ of subdisks of a disk $D$. We say the collection has Property $Z$ (in $D$ ) if (i) the interiors of the $D_{i}$ 's are mutually exclusive, (ii) no two of the $D_{i}$ 's intersect in a disconnected set, and (iii) $\cup D_{i}=D$. If the collection has Property $Z$ and in addition each $D_{i}$ is normally situated in $D$ (that is, each $D_{i}$ is either contained in Int $(D)$ or intersects $\mathrm{Bd}(D)$ in an arc) then we say the collection has Property $Z^{\prime}$. We say the collection has Property $Z(\varepsilon)$ or Property $Z^{\prime}(\varepsilon)$ where $\varepsilon>0$ if the collection has Property $Z$ or Property $Z^{\prime}$ and in addition each $D_{i}$ has diameter less than $\varepsilon$.

2. Some simple lemmas on isotopies and homeomorphisms. Most of the isotopies and homeomorphisms which we construct in this paper and in [13] and which we 
claim are pwl can be shown to be pwl by the following proposition which is stated here without proof. It can be proved by using the lemmas of [25, Chapter 1] to imitate in higher dimensions proofs like those in $[2, \S 1]$. Recall that a polyhedron can be infinite, that is noncompact.

Proposition 2.1. Suppose that $h$ is a homeomorphism of a polyhedron $K$ onto a closed subset of a polyhedron $L$ so that $h$ is $\mathrm{pwl}$ on each element of a locally finite collection of compact subpolyhedra whose union is $K$.

Then $h$ is pwl.

LEMma 2.1. Suppose that $B_{1}^{n}$ and $B_{2}^{n}$ are (pwl) n-cells and $h$ is a (pwl) homeomorphism of $\mathrm{Bd}\left(B_{1}^{n}\right)$ onto $\mathrm{Bd}\left(B_{2}^{n}\right)$. Then there is an extension of $h$ to a (pwl) homeomorphism of $B_{1}^{n}$ onto $B_{2}^{n}$.

Proof. See [25, Lemma 10] for a proof of the pwl version. The topological version is proved similarly.

LEMMA 2.2. Suppose that $\Delta^{n}$ is a $n$-simplex with barycenter $b$, and suppose that $h$ is a pwl homeomorphism of $\Delta^{n}$ onto itself such that $h \mid \operatorname{Bd}\left(\Delta^{n}\right)=I$.

Then there is a pwl isotopy $H_{t}(0 \leqq t \leqq 1)$ of $\Delta^{n}$ onto itself such that $H_{t} \mid \operatorname{Bd}\left(\Delta^{n}\right)=I$ and $H_{1}=h$.

Proof. A proof of this lemma can be found in [25]; however, we repeat the proof in order to make several observations about the constructed isotopy.

There is no loss in assuming that $\Delta^{n} \subset E^{n}=E^{n} \times 0 \subset E^{n} \times E^{1}=E^{n+1}$. Consider the prism $\Delta^{n} \times[0,1]$ in $E^{n+1}$. Define a level preserving pwl homeomorphism $H$ of $\Delta^{n} \times[0,1]$ onto itself as follows. First set $H\left|\operatorname{Bd}\left(\Delta^{n}\right) \times[0,1]=I, H\right| \Delta^{n} \times 0=I$, $H \mid \Delta^{n} \times 1=(h, 1)$, and $H \mid b \times \frac{1}{2}=I$. Then define $H$ on the rest of $\Delta^{n} \times[0,1]$ by the join of the two maps $H \mid \operatorname{Bd}\left(\Delta^{n} \times[0,1]\right)$ and $H \mid b \times \frac{1}{2}$.

The isotopy $H_{t}$ is defined so that $H(y, t)=\left(H_{t}(y), t\right)\left(y \in \Delta^{n}, t \in[0,1]\right)$.

COROllaRY $2.2_{\mathrm{a}}$. If $K$ is a polyhedron (possibly empty) in $\mathrm{Bd}\left(\Delta^{n}\right)$ such that $h \mid b K=I$ then $H_{t} \mid b K=I$.

Proof. If $K$ is empty the problem reduces to showing that $H=I$ on $b \times[0,1]$. But $H \mid b \times \mathrm{Bd}([0,1])=I$ and $H \mid b \times \frac{1}{2}=I$ so $H \mid b \times[0,1]=I$ by the join construction.

Suppose $K$ is not empty. Let $y$ be a point of $K$. Now $H=I$ on $(b y) \times 1$ and on the part of $(b y) \times[0,1]$ not in the 2-simplex $\left(b \times \frac{1}{2}\right)((b y) \times 1)$. But $H \mid\left(b \times \frac{1}{2}\right)((b y) \times 1)$ is the join of two identity maps and so is the identity.

In a similar fashion one can show

COROLlaRY $2.2_{\mathrm{b}}$. If $K$ is a polyhedron in $\mathrm{Bd}\left(\Delta^{n}\right)$ such that $h$ leaves each segment by invariant where $y$ is a point of $K$, then $H_{t}$ leaves each segment by invariant.

COROLlary 2.2. If $\varepsilon>0$ there is a $\delta>0$ such that $d(h, I)<\delta$ implies that $H_{t}$ is an $\varepsilon$-isotopy. 
Proof. Suppose this were not true. Then there would be a positive number $\eta$, there would be a sequence of homeomorphisms $h_{i}$ of $\Delta^{n}$ onto itself such that $h_{i} \mid \operatorname{Bd}\left(\Delta^{n}\right)=I$ and $h_{i} \rightarrow I$, and there would be a sequence of level preserving homeomorphisms $H^{i}$ of $\Delta^{n} \times[0,1]$ onto itself constructed from the $h_{i}$ 's by the join construction such that each $H^{i}$ moved some point by more than $\eta$. But since $\Delta^{n} \times[0,1]$ is compact this would imply that $H^{i} \nrightarrow I$ which contradicts the fact that the limit of the join of two maps is the join of the limits.

LEMMA 2.3. Suppose that $B^{n}$ is $a$ (pwl) $n$-cell and $h$ is $a$ (pwl) homeomorphism of $B^{n}$ onto itself such that $h \mid \mathrm{Bd}\left(B^{n}\right)=I$.

Then there is $a(\mathrm{pwl})$ isotopy $H_{t}(0 \leqq t \leqq 1)$ of $B^{n}$ onto itself such that $H_{t} \mid \mathrm{Bd}\left(B^{n}\right)=I$ and $H_{1}=h$.

Furthermore if $p \in \operatorname{Int}\left(B^{n}\right)$ and $h(p)=p$ then $H_{t}$ can be constructed so that $H_{t} \mid p=I$.

Proof. We prove the pwl version of the lemma. See [1] for a proof of the topological version.

Define a pwl homeomorphism $\phi$ of $B^{n}$ onto an $n$-simplex $\Delta^{n}$ which takes $p$ onto the barycenter $b$ of $\Delta^{n}$. Now $h$ induces $h^{\prime}=\phi h \phi^{-1}: \Delta^{n} \rightarrow \Delta^{n}$. Apply Lemma 2.2 to find a pwl isotopy $H_{t}^{\prime}(0 \leqq t \leqq 1)$ of $\Delta^{n}$ onto itself such that $H_{t}^{\prime} \mid \operatorname{Bd}\left(\Delta^{n}\right)=I$ and $H_{1}^{\prime}=h^{\prime}$. Set $H_{t}=\phi^{-1} H_{t}^{\prime} \phi$.

If $h(p)=p$ then $h^{\prime}(b)=b$ so by Corollary $2.2_{\mathrm{a}}, H_{t}^{\prime} \mid b=I$ and thus $H_{t} \mid p=I$.

With the aid of Corollary $2.2_{\mathrm{c}}$ we obtain the following lemma by imitating the previous construction. See also [17].

LeMma 2.4. Suppose that $B^{n}$ is $a$ (pwl) $n$-cell and $\varepsilon>0$.

There is $a \delta>0$ such that if $h$ is $a$ (pwl) $\delta$-homeomorphism of $B^{n}$ onto itself so that $h \mid \mathrm{Bd}\left(B^{n}\right)=I$, then there is $a$ (pwl) $\varepsilon$-isotopy $H_{t}(0 \leqq t \leqq 1)$ of $B^{n}$ onto itself so that $H_{t} \mid \mathrm{Bd}\left(B^{n}\right)=I$ and $H_{1}=h$.

The next lemma shows that certain isotopies of pwl $n$-cells $(n \leqq 3)$ can be approximated by pwl isotopies.

LemMa 2.5. Suppose that $B^{n}(n \leqq 3)$ is a pwl $n$-cell, $H_{t}(0 \leqq t \leqq 1)$ is an isotopy of $B^{n}$ onto itself such that $H_{t} \mid \mathrm{Bd}\left(B^{n}\right)=I$ and $H_{1}$ is a pwl homeomorphism, and $\varepsilon>0$.

Then there is a pwl isotopy $H_{t}^{\prime}(0 \leqq t \leqq 1)$ of $B^{n}$ onto itself such that $H_{t}^{\prime} \mid \mathrm{Bd}\left(B^{n}\right)=I$, $H_{1}^{\prime}=H_{1}$, and for each $t, d\left(H_{t}, H_{t}^{\prime}\right)<\varepsilon$.

Proof. Choose $\delta$ from Lemma 2.4 for the substitution $B^{n} \rightarrow B^{n}$ and $\varepsilon / 3 \rightarrow \varepsilon$. Let $0=t_{0}<t_{1}<\cdots<t_{m}=1$ be numbers such that for each $i, H_{t}\left(t_{i} \leqq t \leqq t_{i+1}\right)$ is a $\delta / 3$-isotopy of $B^{n}$.

Use [2, Theorem 3] or a lower dimensional version of it to find for each $i$ $(1 \leqq i \leqq m-1)$ a pwl homeomorphism $h_{i}$ of $B^{n}$ such that $d\left(h_{i}, H_{t_{i}}\right)<\delta / 3$ and $h_{i} \mid \operatorname{Bd}\left(B^{n}\right)=I$. Set $h_{0}=I$ and $h_{m}=H_{1}$.

From the inequality $d\left(h_{i}, h_{i+1}\right) \leqq d\left(h_{i}, H_{t_{i}}\right)+d\left(H_{t_{i}}, H_{t_{i+1}}\right)+d\left(H_{t_{i+1}}, h_{i+1}\right)$ we find that $d\left(h_{i}, h_{i+1}\right)=d\left(h_{i+1} h_{i}^{-1}, I\right)<\delta$ for each $i$. Thus from Lemma 2.4 there is for 
each $i(0 \leqq i \leqq m-1)$ a pwl $\varepsilon / 3$-isotopy $H_{t}^{i}(0 \leqq t \leqq 1)$ of $B^{n}$ onto itself such that $H_{t}^{i} \mid \operatorname{Bd}\left(B^{n}\right)=I$ and $H_{1}^{i}=h_{i+1} h_{i}^{-1}$.

Define $H_{t}^{\prime}$ by the rule $H_{0}^{\prime}=I$ and $H_{t}^{\prime}=H_{\left(t-t_{i}\right) /\left(t_{t+1}-t_{t}\right)}^{i} H_{t_{t}}^{\prime}=H_{\left(t-t_{t}\right) /\left(t_{t+1}-t_{t}\right.}^{i} h_{i}$ $\left(t_{i} \leqq t \leqq t_{i+1}\right)$. From the triangle inequality we find that $d\left(H_{t}^{\prime}, H_{t}\right)<\varepsilon$ for each $t$. The restriction $H_{t}^{\prime} \mid \mathrm{Bd}\left(B^{n}\right)=I$, and $H_{1}^{\prime}=\left(h_{m} h_{m-1}^{-1}\right) h_{m-1}=h_{m}=H_{1}$.

By employing Lemma 2.5 to appropriately alter the proofs of [22, Corollary 1 to Theorem 1], [5, Theorem 7.2] and making other small modifications we obtain the following two lemmas.

LEMma 2.6. Suppose that $D$ is a polyhedral disk in $E^{3}, E$ is a polyhedral subdisk of $D$ whose intersection with $\mathrm{Bd}(D)$ is an arc $A$, and $O$ is an open polyhedron containing $(D-E) \cup(\mathrm{Bd}(E) \cap \operatorname{Int}(D))$.

Then there is a pwl isotopy $H_{t}(0 \leqq t \leqq 1)$ of $E^{3}$ onto itself which is the identity except in $O$ such that $H_{1}(D)=E$.

LEMMA 2.7. Suppose that $S$ is a polyhedral 2-sphere in $E^{3}, D$ and $E$ are polyhedral disks which span $S$ from inside and have a common boundary on $S$, and $h$ is a pwl homeomorphism of $D$ onto $E$ which is the identity on $\mathrm{Bd}(D)=\mathrm{Bd}(E)$.

Then there is a pwl isotopy $H_{t}(0 \leqq t \leqq 1)$ of $E^{3}$ onto itself which is the identity except in Int $(S)$ such that $H_{1} \mid D=h$.

LEMmA 2.8. Suppose that $S$ is a polyhedral 2-sphere in $E^{3}$ and $D$ and $E$ are polyhedral disks such that $D \cap S=E \cap S=A$ an arc on $\mathrm{Bd}(D) \cap \mathrm{Bd}(E)$ and $(D-A) \cup(E-A) \subset \operatorname{Int}(S)$.

Then if $h$ is a pwl homeomorphism of $D$ onto $E$ such that $h \mid A=I$, there is a pwl isotopy $H_{t}(0 \leqq t \leqq 1)$ of $E^{3}$ onto itself which is the identity except in Int $(S)$ so that $H_{1} \mid D=h$.

Proof. Let $F$ be a polyhedral disk on $S$ such that $A \subset \operatorname{Bd}(F)$. Both $D \cup F$ and $E \cup F$ are disks whose interiors contain Int $(A)$. Use [12, Lemma 2.4] to find polyhedral 3-cells $C(D)$ and $C(E)$ in $E^{3}$ such that $D \cup F \subset \operatorname{Bd}(C(D)), E \cup F$ $\subset \mathrm{Bd}(C(E)), C(D)-F \subset \operatorname{Int}(S)$, and $C(E)-F \subset \operatorname{Int}(S)$. Let $D^{\prime}$ and $E^{\prime}$ denote the respective polyhedral disks $\mathrm{Bd}(C(D))-\operatorname{Int}(F)$ and $\mathrm{Bd}(C(E))-\operatorname{Int}(F)$.

Extend $h$ to a pwl homeomorphism of $D^{\prime}$ onto $E^{\prime}$ which is the identity on $\operatorname{Bd}\left(D^{\prime}\right)=\operatorname{Bd}\left(E^{\prime}\right)$. Lemma 2.7 now provides a pwl isotopy $H_{t}(0 \leqq t \leqq 1)$ of $E^{3}$ onto itself which is the identity except on Int $(S)$ so that $H_{1} \mid D^{\prime}=h$.

The next lemma is a mild extension of Theorems 7.3 and 7.5 of [5].

LEMMA 2.9. Suppose that $R_{1}, \ldots, R_{i}, \ldots, R_{n}$ are mutually exclusive polyhedral 2-spheres in $E^{3}$ whose diameters are less than $\varepsilon$ :

Suppose that $S_{1}, \ldots, S_{j}, \ldots, S_{m}$ are mutually exclusive compact polyhedral surfaces each of which is in general position with respect to every $R_{i}$.

Suppose that in each $S_{j}-\left(\cup R_{i}\right)$ there is a component $U_{j}$ that lies in $\cap \operatorname{Ext}\left(R_{i}\right)$ so that every component of $S_{j}-U_{j}$ is a disk of diameter less than $\varepsilon$ which is normally situated in $S_{j}$. 
Suppose that $X$ is a compact set in $\left(\bigcap \operatorname{Ext}\left(R_{i}\right)\right) \cup\left(\cup \mathrm{Cl}\left(U_{j}\right)\right)$ each component of which either intersects $\cup \mathrm{Cl}\left(U_{j}\right)$ or contains points whose distances from $\cup R_{i}$ exceed $3 \varepsilon$.

Then there is a pwl $13 \varepsilon$-isotopy $H_{t}(0 \leqq t \leqq 1)$ of $E^{3}$ onto itself which is the identity on $X \cup\left(\cup \mathrm{Cl}\left(U_{j}\right)\right)$ and except in a 3e-neighborhood of $\cup R_{i}$ such that for each $S_{j}$ every component of $H_{1}\left(S_{j}-\mathrm{Cl}\left(U_{j}\right)\right)$ is contained in some $\operatorname{Int}\left(R_{i}\right)$.

Proof. We first consider a special case of the lemma.

No component of any $S_{j}-U_{j}$ intersects $\mathrm{Bd}\left(S_{j}\right)$. In this case a pwl $12 \varepsilon$-isotopy can be constructed in place of the promised 13e-isotopy. This construction is made by simply imitating the steps in the proofs of [5, Theorems 7.3 and 7.5] and using Lemma 2.5 to convert the isotopies promised by [5, Theorems 7.1 and 7.2] to pwl isotopies at each place where one of these theorems is used in the proofs of [5, Theorems 7.3 and 7.5]. The special assumptions we have made concerning the nature of the $U_{j}$ 's make it unecessary to assume as in [5] that the $S_{j}$ 's are 2-spheres.

To finish the proof of the lemma we reduce the general case to the special case just mentioned. There is no loss in assuming that the $R_{i}$ 's have mutually exclusive interiors since any $R_{i}$ can be thrown out if it is contained in the interior of another one. For each $R_{i}$ let $C_{i}$ denote the polyhedral cube whose boundary is $R_{i}$. For each $S_{j}$ let $E_{j 1}, \ldots, E_{j k}, \ldots$ denote the components of $S_{j}-U_{j}$ which intersect $\mathrm{Bd}\left(S_{j}\right)$.

By repeated applications of Lemma 2.6 we construct a pwl $\varepsilon$-isotopy $H_{t}^{1}(0 \leqq t \leqq 1)$ of $E^{3}$ onto itself which is the identity on $X \cup\left(\cup\left(S_{j}-\cup E_{j k}\right)\right)$ and except in an $\varepsilon$-neighborhood of $\bigcup R_{i}$ so that for each $E_{j k}, H_{1}^{1}\left(E_{j k}-\left(E_{j k} \cap \mathrm{Cl}\left(U_{j}\right)\right)\right)$ is contained in some Int $\left(R_{i}\right)$.

Carve away thin mutually exclusive regular neighborhoods of the $H_{1}^{1}\left(E_{j k}\right)$ 's in the $C_{i}$ 's to obtain sets whose closures are polyhedral 3-cells $C_{1}^{\prime}, \ldots, C_{i}^{\prime}, \ldots$ where the boundary $R_{i}^{\prime}$ of each $C_{i}^{\prime}$ contains $\left(\bigcup\left(S_{j}-\left(\bigcup E_{j k}\right)\right)\right) \cap R_{i}$ and is in general position with respect to every $H_{1}^{1}\left(S_{j}\right)$.

For each $j$ let $U_{j}^{\prime}$ denote the subset $U_{j} \cup\left(\cup_{k} H_{1}^{1}\left(E_{j k}\right)\right)$ of the surface $S_{j}^{\prime}=H_{1}^{1}\left(S_{j}\right)$. Retain $X$, but replace each $R_{i}, S_{j}$, and $U_{j}$ by $R_{i}^{\prime}, S_{j}^{\prime}$, and $U_{j}^{\prime}$ in the statement of the lemma. Now we have a situation which fits the special case so there is a pwl 12ع-isotopy $H_{t}^{2}(0 \leqq t \leqq 1)$ of $E^{3}$ which is the identity on $X \cup\left(\cup \mathrm{Cl}\left(U_{j}^{\prime}\right)\right)$ and except in a $3 \varepsilon$-neighborhood of $\bigcup R_{i}^{\prime}$ such that for each $j$ every component of $H_{1}^{2}\left(S_{j}^{\prime}-\mathrm{Cl}\left(U_{j}^{\prime}\right)\right)$ is contained in some Int $\left(R_{i}^{\prime}\right)$.

The promised isotopy is given by $H_{t}=H_{2 t}^{1}\left(0 \leqq t \leqq \frac{1}{2}\right)$ and $H_{t}=H_{2(t-1 / 2)}^{2} H_{1 / 2}$ $\left(\frac{1}{2} \leqq t \leqq 1\right)$. It is a pwl $13 \varepsilon$-isotopy of $E^{3}$. Since $\bigcup U_{j} \subset \bigcup U_{j}^{\prime}$ and a $3 \varepsilon$-neighborhood of $\bigcup R_{i}^{\prime}$ is contained in a $3 \varepsilon$-neighborhood of $\bigcup R_{i}, H_{t}$ is the identity on $X \cup$ $\left(\bigcup \mathrm{Cl}\left(U_{j}\right)\right)$ and except in a $3 \varepsilon$-neighborhood of $\bigcup R_{i}$. Each Int $\left(R_{i}^{\prime}\right) \subset \operatorname{Int}\left(R_{i}\right)$ so every component of a $H_{1}\left(S_{j}-\mathrm{Cl}\left(U_{j}\right)\right)$ is contained in some Int $\left(R_{i}\right)$.

\section{Small isotopies of approximating disks into themselves.}

Lemma 3.1. Suppose that $D$ is a polyhedral disk, $R$ is a polyhedron in $\mathrm{Bd}(D)$, $f$ is a homeomorphism of $D$ into $E^{3}$, and $\varepsilon>0$. 
There is a $\delta>0$ such that if $f^{\prime}$ is a pwl homeomorphism of $D$ into $E^{3}$ where $d\left(f, f^{\prime}\right)<\delta, h$ is a pwl $\delta$-homeomorphism of $f^{\prime}(D)$ onto itself which is the identity on $f^{\prime}(R)$, and $L$ is a polyhedron in $E^{3}$ whose intersection with $f^{\prime}(D)$ is contained in $f^{\prime}(R)$, then there is a pwl $\varepsilon$-isotopy $H_{t}(0 \leqq t \leqq 1)$ of $E^{3}$ onto itself which is the identity on $L$ such that $H_{1} \mid f^{\prime}(D)=h$.

Proof. First consider a special case.

The subpolyhedron $R=\mathrm{Bd}(D)$. In this case choose $\delta$ so that $3 \delta$ is subject to the restrictions on $\delta$ in Lemma 2.4 for the substitution $f(D) \rightarrow B^{n}, \varepsilon / 6 \rightarrow \varepsilon$.

Let $f^{\prime}$ be a pwl homeomorphism of $D$ into $E^{3}$ such that $d\left(f, f^{\prime}\right)<\delta$, let $h$ be a pwl $\delta$-homeomorphism of $f^{\prime}(D)$ onto itself which is the identity on $f^{\prime}(\operatorname{Bd}(D))$, and let $L$ be a polyhedron in $E^{3}$ which misses $f^{\prime}(\operatorname{Int}(D))$. Both $f^{\prime} f^{-1}$ and $f\left(f^{\prime}\right)^{-1}$ are $\delta$-homeomorphisms so $\left(f\left(f^{\prime}\right)^{-1}\right) h\left(f^{\prime} f^{-1}\right)$ is a $3 \delta$-homeomorphism of $f(D)$ onto itself which is the identity on $f(\operatorname{Bd}(D))$.

From Lemma 2.4 there is an $\varepsilon / 6$-isotopy $H_{t}^{0}(0 \leqq t \leqq 1)$ of $f(D)$ onto itself which is the identity on $f(\operatorname{Bd}(D))$ such that $H_{1}^{0}=\left(f\left(f^{\prime}\right)^{-1}\right) h\left(f^{\prime} f^{-1}\right)$. Define an isotopy $H_{t}^{1}(0 \leqq t \leqq 1)$ of $f^{\prime}(D)$ onto itself by $H_{t}^{1}=\left(f^{\prime} f^{-1}\right) H_{t}^{0}\left(f\left(f^{\prime}\right)^{-1}\right)$. We have $H_{0}^{1}=I$, $H_{1}^{1}=h$, and since the track of a point of $f(D)$ under $H_{t}^{0}\left(f\left(f^{\prime}\right)^{-1}\right)$ has diameter less than $\varepsilon / 6$ the track of a point of $f^{\prime}(D)$ under $H_{t}^{1}$ has diameter less than $\varepsilon / 6+2 \delta<\varepsilon / 3$.

From [12, Lemma 2.4] there are polyhedral 3-cells $C_{0}$ and $C_{1}$ in $E^{3}$ such that $C_{0} \cap C_{1}=\operatorname{Bd}\left(C_{0}\right) \cap \operatorname{Bd}\left(C_{1}\right)=f^{\prime}(D)$ and $\left(C_{0} \cup C_{1}\right) \cap L \subset f^{\prime}(\operatorname{Bd}(D))$. From [10] we find embeddings $\lambda_{e}: \mathrm{Bd}\left(C_{e}\right) \times[0,1] \rightarrow C_{e}(e=0,1)$ so that for each point $y$ of $\mathrm{Bd}\left(C_{e}\right), \lambda_{e}(y, 0)=y$. We assume that each $\lambda_{e}(y \times[0,1])$ has diameter less than $\varepsilon / 6$.

Set $H_{t}^{1}=I$ on $\mathrm{Bd}\left(C_{0}\right) \cup \mathrm{Bd}\left(C_{1}\right)-f^{\prime}(\operatorname{Int}(D))$. Then define $H_{t}^{1}$ on $C_{0} \cup C_{1}$ so that it is the identity except on $\bigcup \lambda_{e}\left(\mathrm{Bd}\left(C_{e}\right) \times[0,1]\right)$ and is given by $H_{t}^{1}\left(\lambda_{e}(y, s)\right)$ $=\left(\lambda_{e}\left(H_{t(1-s)}^{1}(y), s\right)\right)$ there. Now $H_{t}^{1}$ is an $\varepsilon / 3+2 \varepsilon / 6$-isotopy of $C_{0} \cup C_{1}$ which is the identity on $\mathrm{Bd}\left(C_{0} \cup C_{1}\right)$. Unfortunately $H_{t}^{1}$ is not necessarily pwl.

From Lemma 2.4 and [2, Theorem 3] we find an $\varepsilon / 3$-isotopy $H_{t}^{2}(0 \leqq t \leqq 1)$ of $C_{0} \cup C_{1}$ onto itself which is the identity on $\mathrm{Bd}\left(C_{0}\right) \cup \mathrm{Bd}\left(C_{1}\right)$ so that $H_{1}^{2} H_{1}^{1}$ is a pwl homeomorphism of $C_{0} \cup C_{1}$ onto itself. Let $H_{t}^{3}$ denote the $\varepsilon$-isotopy of $C_{0} \cup C_{1}$ given by $H_{t}^{3}=H_{2 t}^{1}\left(0 \leqq t \leqq \frac{1}{2}\right)$ and $H_{t}^{3}=H_{2(t-1 / 2)}^{2}\left(\frac{1}{2} \leqq t \leqq 1\right)$. Lemma 2.5 together with [15] provides a pwl $\varepsilon$-isotopy $H_{t}(0 \leqq t \leqq 1)$ of $C_{0} \cup C_{1}$ onto itself which is the identity on $\mathrm{Bd}\left(C_{0} \cup C_{1}\right)$ so that $H_{1}=H_{1}^{3}$. Extend $H_{t}$ to the rest of $E^{3}$ by setting it equal to the identity outside $C_{0} \cup C_{1}$. Since $L$ fails to meet Int $\left(C_{0} \cup C_{1}\right)$ and since $H_{1}\left|f^{\prime}(D)=H_{1}^{2}\right| f^{\prime}(D)=h, H_{t}$ satisfies the conditions in the conclusion of the special case of the lemma.

Now we prove the general case. Let $\delta^{\prime}$ correspond to $D, f$, and $\varepsilon / 2$ in the special case. Let $\delta$ be a positive number so small that a $3 \delta$-homeomorphism of $f(\operatorname{Bd}(D))$ onto itself which is the identity on $f(R)$ is $\delta^{\prime} / 12$-isotopic to the identity in $f(\operatorname{Bd}(D))$ keeping $f(R)$ fixed.

Let $f^{\prime}$ be a pwl homeomorphism of $D$ into $E^{3}$ such that $d\left(f, f^{\prime}\right)<\delta$, let $h$ be a pwl $\delta$-homeomorphism of $f^{\prime}(D)$ onto itself which is the identity on $f^{\prime}(R)$, and let $L$ be a polyhedron in $E^{3}$ whose intersection with $f^{\prime}(D)$ is contained in $f^{\prime}(R)$. 
The homeomorphism $\left(f\left(f^{\prime}\right)^{-1}\right) h\left(f^{\prime} f^{-1}\right)$ is a $3 \delta$-homeomorphism of $f(D)$ onto itself which is the identity on $f(R)$ so there is a $\delta^{\prime} / 12$-isotopy $H_{t}^{1}(0 \leqq t \leqq 1)$ of $f(\mathrm{Bd}(D))$ onto itself which is the identity on $f(R)$ such that

$$
H_{1}^{1}=\left(f\left(f^{\prime}\right)^{-1}\right) h\left(f^{\prime} f^{-1}\right) \mid f(\operatorname{Bd}(D)) .
$$

Define an isotopy $H_{t}^{2}(0 \leqq t \leqq 1)$ of $f^{\prime}(\mathrm{Bd}(D))$ onto itself by the rule

$$
H_{t}^{2}=\left(f^{-1} f^{\prime}\right) H_{t}^{1}\left(f\left(f^{\prime}\right)^{-1}\right) .
$$

It is a $\delta^{\prime} / 12+2 \delta<\delta^{\prime} / 4$-isotopy of $f^{\prime}(\mathrm{Bd}(D))$ onto itself which is the identity on $f^{\prime}(R)$ such that $H_{1}^{2}=h \mid f^{\prime}(\mathrm{Bd}(D))$.

By several applications of [12, Lemma 2.3] we enlarge $f^{\prime}(D)$ to a polyhedral disk $E$ in $E^{3}$ such that $f^{\prime}(R) \subset \mathrm{Bd}(E), E \cap L \subset f^{\prime}(R)$, and $f^{\prime}(\mathrm{Bd}(D)-R) \subset \operatorname{Int}(E)$. By using [10] to put a bicollar on $f^{\prime}(\operatorname{Bd}(D)-R)$ in Int $(E)$ which has fibers of diameter less than $\delta^{\prime} / 12$ and which tapers out near $f^{\prime}(R)$, and then by imitating a step in the special case we extend $H_{t}^{2}(0 \leqq t \leqq 1)$ to a $\delta^{\prime} / 4+2 \delta^{\prime} / 12=5 \delta^{\prime} / 12$-isotopy of $E$ onto itself which is the identity on $\mathrm{Bd}(E)$.

Let $C_{0}$ and $C_{1}$ be polyhedral 3-cells such that $C_{0} \cap C_{1}=\operatorname{Bd}\left(C_{0}\right) \cap \operatorname{Bd}\left(C_{1}\right)=E$ and $\left(C_{0} \cup C_{1}\right)-E$ misses $L$. By collaring $\mathrm{Bd}\left(C_{0}\right)$ and $\mathrm{Bd}\left(C_{1}\right)$ in $C_{0}$ and $C_{1}$ with collars whose fibers have diameters less than $\delta^{\prime} / 12$ and then repeating a step in the special case we extend $H_{t}^{2}(0 \leqq t \leqq 1)$ to a $7 \delta^{\prime} / 12$-isotopy of $C_{0} \cup C_{1}$ which is the identity on $\mathrm{Bd}\left(C_{0} \cup C_{1}\right)$. As before we use Lemma 2.4 and [2, Theorem 3] to find a $\delta^{\prime} / 12$-isotopy $H_{t}^{3}(0 \leqq t \leqq 1)$ of $C_{0} \cup C_{1}$ onto itself which is the identity on $\mathrm{Bd}\left(C_{0} \cup C_{1}\right) \cup f^{\prime}(\mathrm{Bd}(D))$ such that $H_{1}^{3} H_{1}^{2}$ is a pwl homeomorphism of $C_{0} \cup C_{1}$ onto itself and $H_{1}^{3}\left(f^{\prime}(D)\right)=f^{\prime}(D)$.

Consider the $8 \delta^{\prime} / 12$-isotopy $H_{t}^{4}(0 \leqq t \leqq 1)$ of $C_{0} \cup C_{1}$ onto itself which is given by the rule $H_{t}^{4}=H_{2 t}^{2}\left(0 \leqq t \leqq \frac{1}{2}\right)$ and $H_{t}^{4}=H_{2(t-1 / 2)}^{3} H_{1 / 2}^{4}\left(\frac{1}{2} \leqq t \leqq 1\right)$. It is the identity on $\mathrm{Bd}\left(C_{0} \cup C_{1}\right)$ and $H_{1}^{4}$ is a pwl homeomorphism. From Lemma 2.5 and the fact [15] that $C_{0}$ and $C_{1}$ are pwl 3-cells there is a pwl $9 \delta^{\prime} / 12$-isotopy $H_{t}^{5}(0 \leqq t \leqq 1)$ of $C_{0} \cup C_{1}$ onto itself which is the identity on $\mathrm{Bd}\left(C_{0} \cup C_{1}\right)$ such that $H_{1}^{5}=H_{1}^{4}$. Extend $H_{t}^{5}$ to all of $E^{3}$ by setting it equal to the identity off $C_{0} \cup C_{1}$.

Since $H_{1}^{5}\left|f^{\prime}(\operatorname{Bd}(D))=h\right| f^{\prime}(\operatorname{Bd}(D))$ and since $h\left(H_{1}^{5}\right)^{-1}$ is a $9 \delta^{\prime} / 12+\delta<\delta^{\prime}$ homeomorphism of $f^{\prime}(D)$ onto itself which is the identity on $f^{\prime}(\operatorname{Bd}(D))$ there is by the special case of the lemma a pwl $\varepsilon / 2$-isotopy $H_{t}^{6}(0 \leqq t \leqq 1)$ of $E^{3}$ onto itself which is the identity on $L$ so that $H_{1}^{6} \mid f^{\prime}(D)=h\left(H_{1}^{5}\right)^{-1}$.

Set $H_{t}=H_{2 t}^{5}\left(0 \leqq t \leqq \frac{1}{2}\right)$ and $H_{t}=H_{2(t-1 / 2)}^{6} H_{1 / 2}\left(\frac{1}{2} \leqq t \leqq 1\right)$. It is a pwl $\delta^{\prime}+\varepsilon / 2$ $<\varepsilon$-isotopy of $E^{3}$ onto itself which is the identity on $L$, and $H_{1} \mid f^{\prime}(D)$ $=H_{1}^{6} H_{1}^{5} \mid f^{\prime}(D)=h$.

4. Stable graphs on disks. Bing [5] calls a connected planar graph stable if each homeomorphism between two of its images in a 2-sphere can be extended to a homeomorphism of the 2-sphere onto itself. In [5, \$9] he establishes results concerning stable graphs on spheres. With a few minor changes in the proofs of these 
theorems one can obtain the following lemmas. Recall that Property $Z$ is defined in the Introduction.

Lemma 4.1. Suppose that $D$ is a disk and $\varepsilon>0$.

There is $a \delta>0$ such that if $G^{\prime}$ is a finite graph in $D$ which contains $\mathrm{Bd}(D)$ and is such that each component of $D-G^{\prime}$ has diameter less than $\delta$, then there is a subgraph $G$ of $G^{\prime}$ which also contains $\mathrm{Bd}(D)$ so that $G=\bigcup \operatorname{Bd}\left(D_{i}\right)$ where $D_{1}, \ldots, D_{n}$ is a collection of subdisks of $D$ with Property $Z(\varepsilon)$.

Lemma 4.2. Suppose that $D$ is a disk and $D_{1}, \ldots, D_{n}$ is a collection of subdisks which has Property $Z$.

Then $G=\bigcup \mathrm{Bd}\left(D_{i}\right)$ is connected, $G_{O}=\mathrm{Cl}(G-\mathrm{Bd}(D))$ is connected, and no Bd $\left(D_{i}\right)$ separates $G$.

Lemma 4.3. Suppose that $D$ is a (polyhedral) disk and $\varepsilon>0$.

There is $a \delta>0$ such that if $D_{1}, \ldots, D_{n}$ is a collection of (polyhedral) subdisks of $D$ with Property $Z(\delta)$, and if $G$ denotes the graph $\cup \mathrm{Bd}\left(D_{i}\right)$, then any (pwl) $\varepsilon$ homeomorphism $h$ of $G$ into $D$ which takes $\mathrm{Bd}(D)$ onto $\mathrm{Bd}(D)$ can be extended to a (pwl) 3e-homeomorphism of $D$ onto itself.

Lemma 4.4. $A$ finite graph $G$ on a disk $D$ is stable if $G=\bigcup \operatorname{Bd}\left(D_{i}\right)$ where $D_{1}, \ldots, D_{n}$ is a collection of disks with Property $Z$.

Furthermore every homeomorphism of such a graph $G$ into $D$ which takes $\mathrm{Bd}(D)$ onto itself can be extended to a homeomorphism of $D$ onto itself.

5. Some special general position intersections. Recall the definition of Property $Q$ which we gave in [12]. One has a 3-manifold $M$ with triangulation $T$ whose $i$-skeleton is $T_{i}$, a disk $D$ in $M$, a tame Sierpinski curve $X$ normally situated in $D$ (the closure of each component of $D-X$ is a disk which either lies in Int $(D)$ or intersects $\mathrm{Bd}(D)$ in an arc), and a positive number $\eta$ such that each component of $D-X$ has diameter less than $\eta$. A set $I(X, D)$ is defined to be the points of $X$ which are not arcwise accessible from $D-X$. One supposes that $D$ misses $T_{0}$ and $D \cap T_{1}$ is a finite collection of points in $I(X, D)$ where 1-simplexes of $T$ pierce $D$. The quadruple $\left(D, X, T_{2}, \eta\right)$ has Property $Q$ if there is an $\eta$-homeomorphism $g$ of $D$ onto a polyhedral disk $E$ in $M$ such that

(i) $g$ is the identity on $X$,

(ii) $E$ is in general position with respect to $T_{2}$, and

(iii) $E \cap T_{2}=X \cap T_{2}=I(X, D) \cap T_{2}$.

Our interest in systems which have Property $Q$ centers on a graph $G_{O}$ which is defined by considering a particular $\eta$-homeomorphism $g$ of $D$ onto a polyhedral disk $E$ which satisfies (i)-(iii). We define $G_{O}$ to be the sum of the components of the general position intersection $E \cap T_{2}$ which contain points of $T_{1}$. In this section we show that if $D^{\prime}$ is a polyhedral disk which is homeomorphically very close to $D$, if $D^{\prime}$ is in general position with respect to $T_{2}$, if the cardinality of $D^{\prime} \cap T_{1}$ is the 
same as the cardinality of $D \cap T_{1}$, and if $G_{O}^{\prime}$ denotes the graph which is the sum of the components of $D^{\prime} \cap T_{2}$ which contain points of $T_{1}$, then there is a homeomorphism $h$ of $G_{O}$ onto $G_{O}^{\prime}$ such that for each simplex $s$ of $T, h\left(s \cap G_{O}\right)=s \cap G_{O}^{\prime}$.

LEMMA 5.1. Suppose that $D$ is a disk in $E^{3}, L$ is a straight line whose intersection with $D$ is a point $p$ where $L$ pierces $D$, and $\varepsilon>0$.

There is $a \delta>0$ such that if $g$ is a $\delta$-homeomorphism of $D$ onto a polyhedral disk $D^{\prime}$ in $E^{3}$, then there is a pwl isotopy $H_{t}(0 \leqq t \leqq 1)$ of $E^{3}$ onto itself which is the identity except in a $\varepsilon$-neighborhood of p so that $H_{1}\left(D^{\prime}\right) \cap L$ is a point $p^{\prime}$ where a neighborhood of $p^{\prime}$ in $H_{1}\left(D^{\prime}\right)$ lies in the plane through $p^{\prime}$ perpendicular to L. (There is a $\delta>0$ such that if $g_{0}$ and $g_{1}$ are $\delta$-homeomorphisms of $D$ onto disjoint polyhedral disks $D_{0}$ and $D_{1}$ in $E^{3}$, then there is a pwl isotopy $H_{t}(0 \leqq t \leqq 1)$ of $E^{3}$ onto itself which is the identity except in an $\varepsilon$-neighborhood of $p$ so that $H_{1}\left(D_{e}\right) \cap L(e=0,1)$ is a point $p_{e}$ where a neighborhood of $p_{e}$ in $H_{1}\left(D_{e}\right)$ lies in the plane through $p_{e}$ perpendicular to $L$.)

Proof. We prove only the case where there are to be two homeomorphisms $g_{0}$ and $g_{1}$.

Let $O$ be an open set of diameter less than $\varepsilon$ which contains $p$ and whose closure misses $\mathrm{Bd}(D)$. Choose subdisks $D_{1}$ and $D_{2}$ of $D$ in $O$ and a polyhedral cylindrical sphere $C$ in $O$ with axis $L$ whose bases miss $D$ so that $p \in \operatorname{Int}\left(D_{2}\right), D_{2} \subset \operatorname{Int}(C)$, $D_{2} \subset \operatorname{Int}\left(D_{1}\right)$, and $C \cap D \subset \operatorname{Int}\left(D_{1}\right)$.

Let $\delta$ be a positive number less than the distance from $D$ to the bases of $C$ and less than each of the numbers $\rho\left(D-D_{1}, C\right), \rho\left(D-D_{2}, L\right)$, and $\rho\left(D_{2}, C\right)$. From [5, $\$ \$ 5$ and 6] we may also assume that $\delta$ is so small that if $g^{\prime}$ is a $\delta$-homeomorphism of $D$ onto a polyhedral disk $D^{\prime}$ in general position with respect to $C$ and if $U^{\prime}$ denotes the component of $D^{\prime}-C$ which contains $\mathrm{Bd}\left(D^{\prime}\right)$, then exactly one component of $\mathrm{Cl}\left(U^{\prime}\right) \cap C$ separates the bases of $C$.

Let $g_{0}$ and $g_{1}$ be $\delta$-homeomorphisms of $D$ onto disjoint polyhedral disks $D_{0}$ and $D_{1}$ in $E^{3}$. Since $D$ is compact there is a positive number $\delta^{\prime}<\delta$ such that $g_{0}$ and $g_{1}$ are $\delta^{\prime}$-homeomorphisms of $D$.

Let $H_{t}^{1}(0 \leqq t \leqq 1)$ be a pwl $\left(\delta-\delta^{\prime}\right)$-isotopy of $E^{3}$ onto itself which is the identity except in $O$ so that $H_{1}^{1}\left(D_{0} \cup D_{1}\right)$ is in general position with respect to $C$. Let $U_{e}(e=0,1)$ denote the component of $H_{1}^{1}\left(D_{e}\right)-C$ which contains $H_{1}^{1}\left(\mathrm{Bd}\left(D_{e}\right)\right)$. Let $E_{e 1}, \ldots, E_{e i}, \ldots(e=0,1)$ denote the disks which are the components of $H_{1}^{1}\left(D_{e}\right)-U_{e}$. The boundary of exactly one $E_{e i}(e=0,1)$, say $E_{e 1}$, separates the bases of $C$.

From Lemma 2.9 there is a pwl isotopy $H_{t}^{2}(0 \leqq t \leqq 1)$ of $E^{3}$ which is the identity on $\mathrm{Cl}\left(U_{0} \cup U_{1}\right)$ and except in $O$ such that each $H_{1}^{2}\left(\operatorname{Int}\left(E_{e i}\right)\right)(e=0,1)$ is contained in Int $(C)$. Each $\mathrm{Bd}\left(E_{e i}\right)=H_{1}^{2}\left(\mathrm{Bd}\left(E_{e i}\right)\right)(e=0,1 ; i>1)$ bounds a disk on $C$ which misses the bases of $C$. By repeated applications of [5, Theorem 7.1] (we use Lemma 2.5 to convert the isotopies promised by the theorem to pwl isotopies) we push the successive $H_{1}^{2}\left(E_{e t}\right)$ 's over to $C-C \cap L$ and then push them slightly outside $C$ and so construct a pwl isotopy $H_{t}^{3}(0 \leqq t \leqq 1)$ of $E^{3}$ which is the identity on 
$U_{0} \cup U_{1} \cup H_{1}^{2}\left(E_{01} \cup E_{11}\right)$ and except in $O$ such that $H_{1}^{3} H_{1}^{2} H_{1}^{1}\left(D_{e}\right)-H_{1}^{2}\left(E_{e 1}\right)$ $\subset \operatorname{Ext}(C) \cap\left(E^{3}-L\right)(e=0,1)$.

Let $E_{01}^{\prime}$ and $E_{11}^{\prime}$ denote the disjoint disks on $C$ whose boundaries are respectively $\mathrm{Bd}\left(E_{01}\right)$ and $\mathrm{Bd}\left(E_{11}\right)$. Each of these disks contains a base of $C$. Two further applications of [5, Theorem 7.1] like those in the last paragraph give us a pwl isotopy $H_{t}^{4}(0 \leqq t \leqq 1)$ of $E^{3}$ which is the identity on

$$
H_{1}^{3} H_{1}^{2} H_{1}^{1}\left(D_{0} \cup D_{1}\right)-H_{1}^{2}\left(\operatorname{Int}\left(E_{01} \cup E_{11}\right)\right)
$$

and except in $O$ so that $H_{1}^{4} H_{1}^{2}\left(E_{e 1}\right)=E_{e 1}^{\prime}(e=0,1)$.

Define $H_{t}$ by the rule $H_{0}=I$ and $H_{t}=H_{4(t-(i-1) / 4)}^{i} H_{(i-1) / 4}(1 \leqq i \leqq 4 ;(i-1) / 4 \leqq t$ $\leqq i / 4)$. This pwl isotopy is the identity except in the $\varepsilon$-set $O$ and $H_{1}\left(D_{e}\right) \cap L(e=0,1)$ is the point $p_{e}=E_{e 1}^{\prime} \cap L$. Since each $E_{e 1}^{\prime}$ contains a base of $C$ a neighborhood of $p_{e}$ $(e=0,1)$ is contained in the plane through $p_{e}$ perpendicular to $L$.

Lemma 5.2 is a simple extension of Lemma 5.1 and we omit the proof.

LemMA 5.2. Suppose that $T$ is a rectilinear triangulation of $E^{3}$ with $i$-skeleton $T_{\mathfrak{i}}$, $D$ is a disk in $E^{3}$ such that $D \cap T_{1}$ is a finite collection of points $p_{1}, \ldots, p_{k}, \ldots$ where 1-simplexes of $T$ pierce $D$, and $\varepsilon>0$.

There is $a \delta>0$ such that if $g$ is a $\delta$-homeomorphism of $D$ onto a polyhedral disk $D^{\prime}$ in general position with respect to $T_{2}$, then there is a pwl isotopy $H_{t}(0 \leqq t \leqq 1)$ of $E^{3}$ onto itself which is the identity except in mutually exclusive $\varepsilon$-neighborhoods $O_{1}, \ldots, O_{k}, \ldots$ of the $p_{k}$ 's so that $H_{1}\left(D^{\prime}\right)$ is in general position with respect to $T_{2}$ and $H_{1}\left(D^{\prime}\right) \cap T_{1}$ is a finite collection of points $p_{1}^{\prime}, \ldots, p_{k}^{\prime}, \ldots$ where each $p_{k}^{\prime} \in O_{k}$. (There is a $\delta>0$ such that if $g_{0}$ and $g_{1}$ are $\delta$-homeomorphisms of $D$ onto disjoint polyhedral disks $D_{0}$ and $D_{1}$ in general position with respect to $T_{2}$, then there is a pwl isotopy $H_{t}(0 \leqq t \leqq 1)$ of $E^{3}$ onto itself which is the identity except in mutually exclusive e-neighborhoods $O_{1}, \ldots, O_{k}, \ldots$ of the $p_{k}$ 's so that $H_{1}\left(D_{0} \cup D_{1}\right)$ is in general position with respect to $T_{2}$ and $H_{1}\left(D_{e}\right) \cap T_{1}(e=0,1)$ is a finite collection of points $p_{e 1}, \ldots, p_{e k}, \ldots$ where each $p_{e k} \in O_{k}$.)

LEMmA 5.3. Suppose that $T$ is a rectilinear triangulation of $E^{3}$ with $i$-skeleton $T_{i}$, $D$ is a disk in $E^{3}, X$ is a tame Sierpinski curve normally situated in $D$, and $\eta$ is a positive number such that $\left(D, X, T_{2}, \eta\right)$ has Property $Q$.

Let $G_{O}$ denote the finite graph in $I(X, D)$ which consists of the components of $D \cap T_{2}$ which intersect $T_{1}$, and let $G$ denote the graph $G_{O} \cup \mathrm{Bd}(D)$.

Suppose $\varepsilon>0$, and suppose $G_{O} \cap \mathrm{Bd}(D)$ contains at least two points.

There is $a \delta>0$ such that if $g$ is a $\delta$-homeomorphism of $D$ onto a polyhedral disk $D^{\prime}$ in general position with respect to $T_{2}$ so that the cardinality of $D^{\prime} \cap T_{1}$ is the same as the cardinality of $D \cap T_{1}$, then there is a homeomorphism $\pi$ of $G$ onto a finite graph $G^{\prime}=G_{O}^{\prime} \cup \mathrm{Bd}\left(D^{\prime}\right)$ where $G_{O}^{\prime}$ is the sum of the components of $D^{\prime} \cap T_{2}$ which intersect $T_{1}$ so that

1. for each point $p$ of $D \cap T_{1}, \pi(p)$ is a point of $T_{1}$ which is contained in an $\varepsilon$-neighborhood of $p$, and $\pi$ takes no other points of $G$ into $T_{1}$, 
2. for each 2-simplex $\Delta$ of $T$ and each component $t$ of $G_{O} \cap \Delta, \pi(t)$ is an arc component of $G_{O}^{\prime} \cap \Delta$ that lies in an $\varepsilon$-neighborhood of $t$, and

3. for each arc $s$ in $\mathrm{Bd}(D)$ which is the closure of a component of $\mathrm{Bd}(D)-G_{O}$, $\pi(s)$ is the closure of a component of $\mathrm{Bd}\left(D^{\prime}\right)-G_{O}^{\prime}$ and it lies in an $\varepsilon-n e i g h b o r h o o d$ of $s$.

Proof. Choose $\varepsilon_{1}<\varepsilon$ so small that for each 2-simplex $\Delta$ of $T, \varepsilon_{1}$-neighborhoods of the components of $G_{O} \cap \Delta$ are mutually exclusive sets which miss $T_{0}$, for each 2-simplex $\Delta$ of $T$ and each component $t$ of $G_{O} \cap \Delta$ an $\varepsilon_{1}$-neighborhood of $t$ intersects $T_{2}-\operatorname{Int}(\Delta)$ in at least as many components as there are endpoints of $t$ on $\operatorname{Bd}(\Delta)$, and for each point $q$ of $G_{O}$ on $\mathrm{Bd}(D)$ there is an arc $s(q)$ on $\mathrm{Bd}(D)$ of diameter less than $\varepsilon / 2$ which contains the intersection of $\mathrm{Bd}(D)$ with an $\varepsilon_{1}$-neighborhood of $q$. By requiring $\varepsilon_{1}$ to be very small we may assume that the $s(q)$ 's are mutually exclusive.

Since 1-simplexes of $T$ pierce $D$ at the points of $D \cap T_{1}$ it follows that if $\delta$ is sufficiently small and $g$ is a $\delta$-homeomorphism of $D$ onto a disk $D^{\prime}$ of the type described in the hypothesis of the lemma there will have to be a point $p^{\prime}$ of Int $\left(D^{\prime}\right) \cap T_{1}$ in an $\varepsilon_{1}$-neighborhood of each point $p$ of $D \cap T_{1}$. Hence we will be able to define $\pi$ on $D \cap T_{1}$ so that Condition 1 is satisfied.

Let $\Delta$ be a 2-simplex of $T$ and let $t_{1}, \ldots, t_{i}, \ldots$ denote the arcs which are the components of $G_{O} \cap \Delta$. We show what restrictions to place on $\delta$ so that we will be able to define $\pi$ on $G_{O} \cap \Delta$.

Let $\tau$ be a 3-simplex of $T$ which has $\Delta$ as a face, and let $U$ be a connected open set in $E^{3}$ containing $\Delta$ so that $U-\operatorname{Bd}(\tau)$ has exactly two components, $U_{-1}$ and $U_{1}$. For each arc $t_{i}$ let $t_{a i}$ be an arc in $U \cap G_{O} \cap \operatorname{Bd}(\tau)$ which contains a neighborhood of $t_{i}$ in $G_{O} \cap \operatorname{Bd}(\tau)$. We assume that the $t_{a i}$ 's are mutually exclusive and are contained in $\varepsilon_{1}$-neighborhoods of the $t_{i}$ 's. Each $t_{a i}-t_{i}$ misses $\Delta$. Let $G_{a}$ denote the graph $\cup t_{a i}$.

Use [12, Lemma 3.1] and the fact that $G_{a} \subset I(X, D)$ to construct an embedding $\phi$ of $G_{a} \times[-1,1]$ into $U \cap D$ so that

1. for each point $y$ of $G_{a}, \phi(y, 0)=y, \phi(y \times[-1,1]) \subset \mathrm{Bd}(D) \cap s(y)$ if $y \in \dot{\mathrm{Bd}}(D)$, and $\phi(y \times[-1,1])$ misses $\operatorname{Bd}(D)$ if $y \in \operatorname{Int}(D)$,

2. for each $t_{a i}$ and each endpoint $y$ of $t_{a i}, \phi(y \times[-1,1])$ misses $\Delta$ if $y$ misses $\Delta$ and $\phi(y \times[-1,1])$ misses $T_{2}-\operatorname{Int}(\Delta)$ if $y \in \operatorname{Int}(\Delta)$,

3. each $\phi\left(t_{a i} \times[-1,1]\right)$ is contained in an $\varepsilon_{1}$-neighborhood of $t_{i}$,

4. $\phi\left(G_{a} \times[-1,1]\right) \cap\left(G_{O} \cap \operatorname{Bd}(\tau)\right)=G_{a}$,

5. $\phi\left(G_{a} \times e\right) \subset I(X, D) \cap U_{e}(e=-1,1)$.

Here are the restrictions on $\delta$ which will enable us to define $\pi$ on $G_{a} \cap \Delta$ : (1) $\delta<\rho\left(\phi\left(G_{a} \times[-1,1]\right), E^{3}-U\right),(2) \delta<\rho\left(\phi\left(G_{a} \times e\right), E^{3}-U_{e}\right)(e=-1,1)$, (3) for each $t_{a i}$ and each endpoint $y$ of $t_{a i}, \delta<\rho\left(\phi(y \times[-1,1]), T_{2}-\operatorname{Int}(\Delta)\right)$ if $y \in \operatorname{Int}(\Delta)$ and $\delta<\rho(\phi(y \times[-1,1]), \Delta)$ if $y \notin \Delta$, and (4) for each $t_{a i}$ a $\delta$-neighborhood of $\phi\left(t_{a i} \times[-1,1]\right)$ is contained in an $\varepsilon_{1}$-neighborhood of $t_{a i}$. 
We choose $\delta$ subject to restrictions like those in the preceding paragraph for each 2-simplex of $T$ and subject to the restriction mentioned in the second paragraph of the proof. Further we assume that $\delta$ is sufficiently small so that for each arc $s$ with endpoints $q_{1}$ and $q_{2}$ where $s$ is the closure of a component of $\mathrm{Bd}(D)-G_{O}$, a $\delta$-neighborhood of $s \cup s\left(q_{1}\right) \cup s\left(q_{2}\right)$ is contained in an $\varepsilon$-neighborhood of $s$.

Let $g$ be a $\delta$-homeomorphism of $D$ onto a polyhedral disk $D^{\prime}$ in general position with respect to $T_{2}$ so that the cardinality of $D^{\prime} \cap T_{1}$ is the same as the cardinality of $D \cap T_{1}$, and let $G_{O}^{\prime}$ and $G^{\prime}$ be defined as indicated in the hypothesis of the lemma.

From the second paragraph of the proof $\pi$ can be defined to take $D \cap T_{1}$ onto $D^{\prime} \cap T_{1}$ so that for each point $p$ of $D \cap T_{1}, \pi(p)$ is contained in an $\varepsilon_{1}$-neighborhood of $p$. Thus Condition 1 is satisfied in the conclusion of the lemma.

We show how to define $\pi$ on $G_{O} \cap \Delta$.

Consider an arc $t_{i}$ and suppose that it has endpoints $p_{1}(i)$ and $p_{2}(i)$ on $\operatorname{Bd}(\Delta)$. In this case $t_{a i}$ has endpoints $p_{a 1}(i)$ and $p_{a 2}(i)$ in $\mathrm{Bd}(\tau)-\Delta$. From Conditions 2, 3 and 4 on the choice of $\delta, \mathrm{Bd}(\tau)$ separates $g \phi\left(t_{a i} \times-1\right)$ from $g \phi\left(t_{a i} \times 1\right)$ and there is an arc $t_{a i}^{\prime}$ in $g \phi\left(t_{a i} \times[-1,1]\right) \cap \mathrm{Bd}(\tau)$ which runs from a point $p_{a 1}^{\prime}(i)$ of

$$
g \phi\left(p_{a 1}(i) \times[-1,1]\right) \cap(\mathrm{Bd}(\tau)-\Delta)
$$

to a point $p_{a 2}^{\prime}(i)$ of $g \phi\left(p_{a 2}(i) \times[-1,1]\right) \cap(\mathrm{Bd}(\tau)-\Delta)$. The arc $t_{a i}^{\prime}$ is contained in an $\varepsilon_{1}$-neighborhood of $t_{i}$, and since an $\varepsilon_{1}$-neighborhood of $t_{i}$ intersects $\operatorname{Bd}(\tau)$ -Int $(\Delta)$ in at least two components $t_{a i}^{\prime}$ must intersect $\Delta$ in a subarc $t_{i}^{\prime}$ whose endpoints can only be $p_{1}^{\prime}(i)=\pi\left(p_{1}(i)\right)$ and $p_{2}^{\prime}(i)=\pi\left(p_{2}(i)\right)$. Define $\pi$ on $t_{i}$ so that $\pi\left(t_{i}\right)=t_{i}^{\prime}$.

Suppose an arc $t_{i}$ has just one endpoint $p(i)$ on $\mathrm{Bd}(\Delta)$. In this case $t_{a i}$ has one endpoint $p_{a}(i)$ in $\operatorname{Bd}(\tau)-\Delta$ and one endpoint $q(i)$ in Int $(\Delta)$. As before $\operatorname{Bd}(\tau)$ separates $g \phi\left(t_{a i} \times-1\right)$ from $g \phi\left(t_{a i} \times 1\right)$ and there is an arc $t_{a i}^{\prime}$ which runs from a point $p_{a}^{\prime}(i)$ in $g \phi\left(p_{a}(i) \times[-1,1]\right) \cap(\mathrm{Bd}(\tau)-\Delta)$ to a point $q^{\prime}(i)$ in $g \phi(q(i) \times[-1,1])$ $\cap$ Int $(\Delta)$. This arc is contained in an $\varepsilon_{1}$-neighborhood of $t_{i}$ and it intersects $\operatorname{Bd}(\Delta)$ only in $p^{\prime}(i)=\pi(p(i))$ so it contains a subarc $t_{i}^{\prime}$ in $\Delta$ which runs from $p^{\prime}(i)$ to $q^{\prime}(i)$. Define $\pi$ on $t_{i}$ so that $\pi\left(t_{i}\right)=t_{i}^{\prime}$.

Since the arcs $t_{i}^{\prime}$ account for all the components of $G_{O}^{\prime} \cap \Delta$ we have defined $\pi$ to take $G_{O} \cap \Delta$ onto $G_{O}^{\prime} \cap \Delta$. For each remaining 2-simplex of $T$ define $\pi$ in the manner just described. Now $\pi$ takes $G_{O}$ onto $G_{O}^{\prime}$ so that Condition 2 is satisfied in the conclusion of the lemma.

From Condition 1 on the construction of $\phi$ each $\pi(q) \in g(s(q))$ where $q \in G_{O}$ $\cap \mathrm{Bd}(D)$. Since the $s(q)$ 's are mutually exclusive we can define $\pi$ on $\mathrm{Bd}(D)$ by slightly adjusting $g \mid \mathrm{Bd}(D)$ so that it takes each $q$ onto $\pi(q)$. If $s$ is the closure of a component of $\mathrm{Bd}(D)-G_{O}$ with endpoints $q_{1}$ and $q_{2}$ then $\pi(s) \subset g\left(s \cup s\left(q_{1}\right) \cup s\left(q_{2}\right)\right)$ which is contained in an $\varepsilon$-neighborhood of $s$ by the choice of $\delta$. Thus Condition 3 is satisfied in the conclusion of the lemma.

6. A construction involving Property $Q$. We describe a construction which is employed in the proofs of Theorem 7.1 here and Theorem 5.1 of [13]. Hypotheses 
are introduced before Lemmas 6.1, 6.4, 6.5, and 6.6. Once introduced a hypothesis is to be kept for the remainder of $\S 6$. The same is to hold for the numbers $\eta, \delta_{1}, \delta$, $\varepsilon_{1}$, and $\varepsilon_{2}$ which appear at various places in $\S 6$.

Consider a polyhedral disk $D$, a homeomorphism $f$ of $D$ into $E^{3}$, a positive number $\eta$, and a rectilinear triangulation $T$ of $E^{3}$ with $i$-skeleton $T_{i}$ and with mesh less than $\eta$. Let $\Delta_{1}, \ldots, \Delta_{j}, \ldots$ denote the 2-simplexes of $T$. From [12, Theorem 6.1] there is a tame Sierpinski curve $X$ normally situated in $D$, and there is an $\eta$-homeomorphism $h$ of $E^{3}$ onto itself which is the identity outside an $\eta$-neighborhood of $f(D)$ so that $\left(h f(D), h(X), T_{2}, \eta\right)$ has Property $Q$. Let $G_{\mathrm{IV}}$ denote the finite graph which consists of $h f(\mathrm{Bd}(D))$ and the components of $h(X) \cap T_{2}$ that meet $T_{1}$. Set $G_{\mathrm{III}}=h^{-1}\left(G_{\mathrm{IV}}\right)$.

From the definition of Property $Q$ there is a homeomorphism $\phi$ of $h f(D)$ onto a polyhedral disk $E$ in general position with respect to $T_{2}$ so that $\phi \mid h(X)=I$ and $E \cap T_{2}=h(X) \cap T_{2}$.

LEMMA 6.1. Let $\delta_{1}$ be a positive number.

There is a positive number $\delta$ so that if $h^{\prime}$ is a homeomorphism of $E^{3}$ for which $d\left(h, h^{\prime}\right)<\delta_{1} / 2$ and $f^{\prime}$ is a homeomorphism of $D$ into $E^{3}$ for which $d\left(f, f^{\prime}\right)<\delta$ then $d\left(h f, h^{\prime} f^{\prime}\right)<\delta_{1}$.

Proof. Suppose the lemma were not true. Then there would be a homeomorphism $h^{\prime}$ such that $d\left(h, h^{\prime}\right)<\delta_{1} / 2$, and there would be a sequence of homeomorphisms $f_{1}, \ldots, f_{n}, \ldots$ of $D$ into $E^{3}$ such that $f_{n} \rightarrow f$ and for each $n, d\left(h f, h^{\prime} f_{n}\right) \geqq \delta_{1}$. But by the continuity of $h^{\prime}, h^{\prime} f_{n} \rightarrow h^{\prime} f$ so there is an $N$ such that $d\left(h^{\prime} f_{n}, h^{\prime} f\right)<\delta_{1} / 2(n \geqq N)$. But then $d\left(h^{\prime} f_{n}, h f\right) \leqq d\left(h^{\prime} f_{n}, h^{\prime} f\right)+d\left(h^{\prime} f, h f\right)<\delta_{1} / 2+\delta_{1} / 2=\delta_{1}$.

Lemma 6.2. Let $\varepsilon_{1}$ be a positive number.

If $\eta$ is sufficiently small then each component of $h f(D)-G_{\mathrm{IV}}$ has diameter less than $\varepsilon_{1}$.

Proof. Suppose $\eta$ is so small that each $3 \eta$-subset of $f(D)$ lies in an $\varepsilon_{1} / 6$-disk which is normally situated in $f(D)$.

Let $x$ and $y$ be a pair of points in $h f(D)-G_{\text {IV }}$ such that $\rho(x, y) \geqq \varepsilon_{1}$. We show that $G_{\text {IV }}$ separates $x$ from $y$. Denote by $Z$ the sum of all 2-simplexes of $T$ whose distances from $x \cup y$ exceed $\varepsilon_{1} / 3$. Since $\eta<\varepsilon_{1} / 3, Z$ separates $x$ from $y$ in $E^{3}$ so $Z \cap h f(D)$ separates $x$ from $y$ in $h f(D)$. Let $Z_{1}$ be a subset of $Z \cap h f(D)$ which is irreducible with respect to separating $x$ from $y$. The unicoherence of $h f(D)$ shows that $Z_{1}$ is connected.

Either $Z_{1}$ fails to intersect $T_{1}$ or it intersects $T_{1}$. In the first case it lies in some 2-simplex of $T$ and so has diameter less than $\eta$; thus $h^{-1}\left(Z_{1}\right)$ has diameter less than $3 \eta$ so it is contained in an $\varepsilon_{1} / 6$-disk that is normally situated in $f(D)$. But then $Z_{1}$ is contained in an $\varepsilon_{1} / 6+2 \eta$ - or $\varepsilon_{1} / 3$-disk that is nurmally situated in $h f(D)$. Such a disk neither separates $h f(D)$ nor intersects $x \cup y$ since $\rho(Z, x \cup y)>\varepsilon_{1} / 3$. We conclude that $Z_{1}$ intersects $T_{1}$. But then $Z_{1}$ intersects $h(I(X, f(D))$. Since $h(A(X, f(D)))$ 
misses $T_{2}$ and separates $h(f(D)-X)$ from $h(I(X, f(D))), Z_{1} \subset G_{\mathrm{IV}}$ so $G_{\mathrm{IV}}$ separates $x$ from $y$.

LEMMA 6.3. Let $\varepsilon_{2}$ be a positive number.

If $\varepsilon_{1}$ is sufficiently small then $G_{\mathrm{III}}$ contains a stable subgraph $G_{\mathrm{I}}$ containing $f(\mathrm{Bd}(D))$ such that $G_{\mathrm{I}}=\bigcup \mathrm{Bd}\left(D_{m}^{\mathrm{I}}\right)$ where $D_{1}^{\mathrm{I}}, \ldots, D_{m}^{\mathrm{I}}, \ldots$ is a collection of subdisks of $f(D)$ which has Property $Z^{\prime}\left(\varepsilon_{2}\right)$.

Proof. Each component of $f(D)-G_{\text {III }}$ is the preimage of a component of $h f(D)-G_{\text {IV }}$ so it has diameter less than $\varepsilon_{1}+2 \eta<2 \varepsilon_{1}$.

Lemma 4.1 together with the fact that $G_{\mathrm{IV}}$ arises from the general position intersection of the polyhedral disk $E$ with $T_{2}$ shows that the ( $D_{m}^{\mathrm{I}}$ )'s can be found provided $2 \varepsilon_{1}$ is subject to the restrictions on $\delta$ in Lemma 4.1 for the substitution $f(D) \rightarrow D$ and $\varepsilon_{2} \rightarrow \varepsilon$.

Set $G_{\mathrm{II}}=h\left(G_{\mathrm{I}}\right)$ and for each $m, D_{m}^{\mathrm{II}}=h\left(D_{m}^{\mathrm{I}}\right)$. Each $D_{m}^{\mathrm{II}}$ has diameter less than $\varepsilon_{2}+2 \eta<2 \varepsilon_{2}$. For each $D_{m}^{\text {II }}$ let $r_{m}$ denote $\mathrm{Cl}\left(\mathrm{Bd}\left(D_{m}^{\mathrm{II}}\right) \cap h f(\operatorname{Int}(D))\right)$. If $D_{m}^{\mathrm{II}} \subset$ $h f(\operatorname{Int}(D))$ then $r_{m}=\mathrm{Bd}\left(D_{m}^{\mathrm{II}}\right)$, and if $D_{m}^{\mathrm{II}}$ intersects $h f(\mathrm{Bd}(D))$ then $r_{m}$ is the arc in $\operatorname{Bd}\left(D_{m}^{\mathrm{II}}\right)$ which spans $h f(\mathrm{Bd}(D))$. Let $G_{\text {IO }}$ and $G_{\text {IIO }}$ denote the respective graphs $\mathrm{Cl}\left(G_{\mathrm{I}} \cap f(\operatorname{Int}(D))\right)$ and $\mathrm{Cl}\left(G_{\mathrm{II}} \cap h f(\operatorname{Int}(D))\right)$. Lemma 4.2 shows that $G_{\text {Io }}$ and $G_{\text {IIO }}$ are connected.

Let $T^{\prime}$ be a subdivision of $T$ in which $h(X) \cap T_{2}$ and $E=\phi h f(D)$ underlie full subcomplexes, and let $T^{\prime \prime}$ be a first derived subdivision of $T^{\prime}$. For each $r_{m}$ define a polyhedron $L\left(r_{m}\right)$ to be the sum of the components of the $\left(N\left(r_{m}, T^{\prime \prime}\right) \cap \Delta_{j}\right)$ 's that contain nondegenerate subsets of $r_{m}$.

Lemma 6.4. Each $L\left(r_{m}\right)$ is a 2-manifold with boundary such that $r_{m} \subset \operatorname{Int}\left(L\left(r_{m}\right)\right)$. If $r_{m}$ is an arc then $L\left(r_{m}\right)$ is a 2-cell, and if $r_{m}$ is a simple closed curve then $L\left(r_{m}\right)$ is an annulus.

Proof. The reader may verify that each $L\left(r_{m}\right)$ is a 2-manifold with boundary which collapses to $r_{m}$ and which is such that $r_{m} \subset \operatorname{Int}\left(L\left(r_{m}\right)\right)$.

If $r_{m}$ is an arc then $L\left(r_{m}\right)$ is a 2-cell since a 2-cell is the only surface which collapses to an arc. Suppose that $r_{m}$ is a simple closed curve. The disk $\phi\left(D_{m}^{\mathrm{II}}\right)$ intersects $L\left(r_{m}\right)$ in exactly $r_{m}$, and it can be fattened up into a polyhedral 3-cell whose boundary contains $L\left(r_{m}\right)$. But then $L\left(r_{m}\right)$ must be an annulus since an annulus is the only planar surface which collapses to a simple closed curve.

Let $T^{m}$ be a first derived subdivision of $T^{\prime \prime}$. Consider the regular neighborhoods $N\left(G_{\text {IIO }}, T^{\prime \prime \prime}\right)$ and for each $m, N\left(r_{m}, T^{m}\right)$. Since $G_{\text {IIO }}$ is connected $N\left(G_{\text {IIO }}, T^{m}\right)$ is a cube-with-handles. Each $N\left(G_{\text {IIO }}, T^{m}\right) \cap L\left(r_{m}\right)$ is a regular neighborhood of $r_{m}$ in Int $\left(L\left(r_{m}\right)\right)$.

LemMa 6.5. Each $L\left(r_{m}\right)$ separates $N\left(G_{\text {IIO }}, T^{m}\right)$ and every $N\left(r_{m}, T^{m}\right)$ into two components. For each $r_{m}$ one of the components $N_{o}\left(r_{m}, T^{\prime \prime}\right)$ of $N\left(r_{m}, T^{m}\right)-L\left(r_{m}\right)$ contains no point of $G_{\mathrm{IIO}}$ and is also a component of $N\left(G_{\mathrm{IIO}}, T^{m}\right)-L\left(r_{m}\right)$. Furthermore the 
$N_{o}\left(r_{m}, T^{m}\right)$ 's are mutually exclusive and are in fact exactly the components of $N\left(G_{\text {IIO }}, T^{m}\right)-\left(\bigcup L\left(r_{m}\right)\right)$.

Proof. Since $E$ underlies a full subcomplex of $T^{\prime}, N\left(E, T^{\prime \prime}\right)$ is a regular neighborhood of $E$ in $E^{3}$. Similarly each $N\left(\phi\left(D_{m}^{\mathrm{I}}\right), T^{\prime \prime}\right)$ is a regular neighborhood of $\phi\left(D_{m}^{\mathrm{I}}\right)$ in $E^{3}$.

Each $L\left(r_{m}\right)$ is two sided so it separates the corresponding $N\left(r_{m}, T^{\prime \prime}\right)$ into two components. Since $r_{m}$ separates $E$ into the two components $\phi\left(D_{m}^{\mathrm{II}}-r_{m}\right)$ and $E-\phi\left(D_{m}^{\mathrm{I}}\right)$, each of the two nonintersecting connected sets $N\left(\phi\left(D_{m}^{\mathrm{II}}-r_{m}\right), T^{m}\right)-L\left(r_{m}\right)$ and $N\left(E-\phi\left(D_{m}^{\mathrm{I}}\right), T^{m}\right)-L\left(r_{m}\right)$ intersects just one of the components of $N\left(r_{m}, T^{m}\right)$ $-\dot{L}\left(r_{m}\right)$. Thus $N\left(E, T^{m}\right)-L\left(r_{m}\right)$ has two components- $N_{O m}\left(E, T^{m}\right)$ which contains $\phi\left(D_{m}^{\mathrm{II}}-r_{m}\right)$ and $N_{U m}\left(E, T^{m}\right)$ which contains $E-\phi\left(D_{m}^{\mathrm{II}}\right)$. This shows that $N\left(r_{m}, T^{m}\right)$ $-L\left(r_{m}\right)$ has exactly two components since it is a subset of $N\left(E, T^{\prime \prime}\right)-L\left(r_{m}\right)$ and can have at most two components. Let these two components be denoted by $N_{o}\left(r_{m}, T^{\prime \prime \prime}\right) \subset N_{O m}\left(E, T^{m}\right)$ and $N_{U}\left(r_{m}, T^{\prime \prime \prime}\right) \subset N_{U m}\left(E, T^{\prime \prime}\right)$. Since $G_{\text {IIo }}-r_{m} \subset\left(E-\phi\left(D_{m}^{\mathrm{II}}\right)\right)$ we see that $N_{o}\left(r_{m}, T^{m}\right)$ is also a component of $N\left(G_{\mathrm{IIO}}, T^{m}\right)-L\left(r_{m}\right)$ and contains no point of $G_{\text {IIO }}-r_{m}$.

No two of the $N_{O m}\left(E, T^{m}\right)$ 's intersect for if they did some $r_{m}$ would fail to separate $E$. Thus the $N_{o}\left(r_{m}, T^{m}\right)$ 's are mutually exclusive, and since

$$
N\left(G_{\text {IIO }}, T^{\prime \prime}\right)=\left(\bigcup N_{O}\left(r_{m}, T^{m}\right)\right) \cup\left(\bigcup L\left(r_{m}\right)\right)
$$

the $N_{o}\left(r_{m}, T^{m}\right)$ 's are the components of $N\left(G_{\text {IIO }}, T^{m}\right)-\left(\bigcup L\left(r_{m}\right)\right)$.

Let $\varepsilon_{3}$ be a positive number. Let $f^{\prime}$ be a pwl homeomorphism of $D$ into $E^{3}$ such that $f^{\prime}(D)$ is in general position with respect to $T_{2}$, the cardinality of $f^{\prime}(D) \cap T_{1}$ is the same as the cardinality of $h f(D) \cap T_{1}$, and $d\left(h f, f^{\prime}\right)<\delta_{1}$. Lemma 5.3 shows that if $\delta_{1}$ is sufficiently small there is a homeomorphism $\pi^{\prime}$ of $G_{\mathrm{IV}}$ onto the graph $G_{\mathrm{IV}}^{\prime}$ that consists of $f^{\prime}(\mathrm{Bd}(D))$ together with the components of $f^{\prime}(D) \cap T_{2}$ that intersect $T_{1}$. The homeomorphism $\pi^{\prime}$ takes $h f(D) \cap T_{1}$ onto $f^{\prime}(D) \cap T_{1}$ and takes each point $p$ of $h f(D) \cap T_{1}$ into an $\varepsilon_{3}$-neighborhood of itself. Further for each 2-simplex $\Delta$ of $T$ and each arc component $t$ of $G_{\mathrm{IV}} \cap \Delta$ that intersects $T_{1}, \pi^{\prime}$ takes $t$ onto a component $t^{\prime}$ of $G_{\mathrm{IV}}^{\prime} \cap \Delta$ that lies in an $\varepsilon_{3}$-neighborhood of $t$. Finally for each arc $s$ which is the closure of a component of $h f(\mathrm{Bd}(D))-G_{\mathrm{IV}}, \pi^{\prime}$ takes $s$ onto an arc $s^{\prime}$ that lies in an $\varepsilon_{3}$-neighborhood of $s$ and is the closure of a component of $f^{\prime}(\operatorname{Bd}(D))-\mathrm{Cl}\left(G_{\mathrm{IV}}^{\prime} \cap f^{\prime}(\operatorname{Int}(D))\right)$.

LEMMA 6.6. Let $\varepsilon_{4}$ be a positive number.

If $\delta_{1}, \varepsilon_{2}$, and $\varepsilon_{3}$ are sufficiently small then $\pi^{\prime} \mid G_{\mathrm{II}}$ can be extended to an $\varepsilon_{4}$-homeomorphism $\pi$ of $h f(D)$ onto $f^{\prime}(D)$ so that for each $D_{m}^{\mathrm{II}}$, Int $\left(N_{o}\left(r_{m}, T^{\prime \prime \prime}\right)\right) \cup \pi\left(r_{m}\right)$ contains a neighborhood of $\pi\left(r_{m}\right)$ in $\pi\left(D_{m}^{\mathrm{II}}\right)$ and so that each $\pi\left(D_{m}^{\mathrm{I}}\right)$ has diameter less than $\varepsilon_{4}$.

Proof. Each $D_{m}^{\mathrm{II}}$ has diameter less than $2 \varepsilon_{2}$ so by requiring that $\varepsilon_{2}<\varepsilon_{4} / 2$ we insure that each $D_{m}^{\mathrm{II}}$ has diameter less than $\varepsilon_{4}$. 
For each $D_{m}^{\mathrm{II}}$ let $O_{m}$ be an open set in $E^{3}$ of diameter less than $\varepsilon_{4}$ which contains $D_{m}^{\mathrm{II}}$. Require $\varepsilon_{3}$ to be so small that an $\varepsilon_{3}$-neighborhood of each $r_{m}$ is contained in $O_{m}$ and does not intersect $\mathrm{Bd}\left(L\left(r_{m}\right)\right)$.

For each $r_{m}$ use [12, Lemma 3.1] and the fact that $I(h(X), h f(D)) \subset E$ to construct a homeomorphism $\lambda_{m}$ of $r_{m} \times[-1,1]$ onto a set $A_{m}$ in $h f(D) \cap \operatorname{Int}\left(N\left(r_{m}, T^{\prime \prime}\right)\right)$ so that $r_{m,-1}=\lambda_{m}\left(r_{m} \times-1\right) \subset \operatorname{Int}\left(N_{o}\left(r_{m}, T^{m}\right)\right), r_{m, 1}=\lambda_{m}\left(r_{m} \times 1\right) \subset \operatorname{Int}\left(N_{U}\left(r_{m}, T^{m}\right)\right)$, and for each point $y$ of $r_{m}, \lambda_{m}(y, 0)=y, \lambda_{m}(y \times[-1,1]) \subset h f(\operatorname{Bd}(D))$ if $y \in h f(\operatorname{Bd}(D))$, and $\lambda_{m}(y \times[-1,1]) \subset h f(\operatorname{Int}(D))$ if $y \in h f(\operatorname{Int}(D))$. We assume that the $\lambda_{m}$ 's are such that each $D_{m}^{m} \cup A_{m} \subset O_{m}$, and for each $\Delta_{j}$ there is a point $y$ of $r_{m}$ such that $\lambda_{m}(y \times[-1,1])$ misses $\Delta_{j}$.

Require $\delta_{1}$ to be so small that for each $D_{m}^{\mathrm{II}}$ a $\delta_{1}$-neighborhood of $D_{m}^{\mathrm{II}} \cup A_{m}$ is contained in $O_{m}$, a $\delta_{1}$-neighborhood of $A_{m}$ is contained in $N\left(r_{m}, T^{\prime \prime \prime}\right)$, a $\delta_{1}$-neighborhood of $r_{m,-1}$ is contained in $N_{o}\left(r_{m}, T^{m}\right)$, and a $\delta_{1}$-neighborhood of $r_{m, 1}$ is contained in $N_{U}\left(r_{m}, T^{m}\right)$. Also require $\delta_{1}$ to be so small that for each 2-simplex $\Delta_{j}$ of $T$ and each $A_{m}$, a $\delta_{1}$-neighborhood of $\Delta_{j}$ does not contain a subset of $A_{m}$ which separates $r_{m,-1}$ from $r_{m, 1}$.

From the conditions on $\varepsilon_{3}$ and $\delta_{1}$ and the fact that $G_{\mathrm{IV}}^{\prime}$ contains all the components of $f^{\prime}(D) \cap T_{2}$ that intersect $T_{1}$, each $r_{m}^{\prime}=\pi^{\prime}\left(r_{m}\right) \subset f^{\prime}\left((h f)^{-1}\left(A_{m}\right)\right) \cap \operatorname{Int}\left(L\left(r_{m}\right)\right)$ and it separates $f^{\prime}\left((h f)^{-1}\left(r_{m,-1}\right)\right) \subset \operatorname{Int}\left(N_{o}\left(r_{m}, T^{\prime \prime}\right)\right)$ from

$$
f^{\prime}\left((h f)^{-1}\left(r_{m, 1}\right)\right) \subset \operatorname{Int}\left(N_{U}\left(r_{m}, T^{m}\right)\right)
$$

in $f^{\prime}\left((h f)^{-1}\left(A_{m}\right)\right)$.

From the last condition on $\delta_{1}$ no component of a $f^{\prime}\left((h)^{-1}\left(A_{m}\right)\right) \cap L\left(r_{m}\right)$ other than $r_{m}^{\prime}$ separates $f^{\prime}\left((h f)^{-1}\left(r_{m,-1}\right)\right)$ from $f^{\prime}\left((h f)^{-1}\left(r_{m, 1}\right)\right)$ in $f^{\prime}\left((h f)^{-1}\left(A_{m}\right)\right)$. Consider the disk $F_{m}$ in $f^{\prime}\left((h f)^{-1}\left(D_{m}^{\mathrm{I}} \cup A_{m}\right)\right)$ whose boundary intersects $f^{\prime}(\operatorname{Int}(D))$ in exactly Int $\left(r_{m}^{\prime}\right)$. From the preceding remark $F_{m} \cap\left(r_{m}^{\prime} \cup \operatorname{Int}\left(N_{o}\left(r_{m}, T^{\prime \prime}\right)\right)\right)$ contains a neighborhood of $r_{m}^{\prime}$ in $F_{m}$. Define $\pi$ on $D_{m}^{\mathrm{II}}$ so that it extends $\pi^{\prime} \mid \operatorname{Bd}\left(D_{m}^{\mathrm{II}}\right)$ and sends $D_{m}^{\text {II }}$ onto $F_{m}$.

The proof that $\pi$ is a homeomorphism is the same as the proof of Lemma 4.4 (see [5, Theorem 9.5]). Since each $D_{m}^{\mathrm{II}} \cup F_{m} \subset O_{m}, \pi$ is an $\varepsilon_{4}$-homeomorphism and each $\pi\left(D_{m}^{\text {II }}\right)$ has diameter less than $\varepsilon_{4}$.

7. Building cartesian products of disks with $[0,1]$. The theorem which follows is a modification of a theorem of Bing's [5, Theorem 3.2]. His proof of that theorem can be used as a rough model for the proof of Theorem 7.1 here. We prefer to use pwl homeomorphisms where Bing used homeomorphisms and rectilinear triangulations of $E^{3}$ where Bing used fences.

THEOREM 7.1. Suppose that $D$ is a polyhedral 2-cell, $f$ is a homeomorphism of $D$ into $E^{3}$, and $\varepsilon>0$.

There is a $\delta>0$ such that if $f_{0}$ and $f_{1}$ are pwl homeomorphisms of $D$ onto disjoint polyhedral disks $D_{0}$ and $D_{1}$ in $E^{3}$ so that $d\left(f, f_{e}\right)<\delta(e=0,1)$, then there is a 
polyhedral stable graph $G$ in $D$ which contains $\mathrm{Bd}(D)$ and there is a pwl homeomorphism $g$ of $G \times[0,1]$ into $E^{3}$ so that

1. each component of $D-G$ has diameter less than $\varepsilon$,

2. for each point $y$ of $G, g(y, e)=f_{e}(y)(e=0,1)$ and the diameter of $g(y \times[0,1])$ is less than $\varepsilon$, and

3. $g(G \times[0,1]) \cap D_{e}=f_{e}(G)(e=0,1)$.

Proof. The proof is broken into five steps. We introduce at various places a $\delta_{1}, \eta, \gamma$, and several $\varepsilon$ 's where the size of each of these numbers is provisional upon the numbers introduced later. We employ the sequence of lemmas in $\$ 6$, but we do not attempt to make the indices of our $\varepsilon$ 's conform to the indices of the $\varepsilon$ 's in $\$ 6$.

Step 1. Locating the graph $G$. Let $\varepsilon_{1}$ be a positive number. From $\S 6$ there is a positive number $\eta<\varepsilon_{1} / 2$, a tame Sierpinski curve $X$ normally situated in $f(D)$, a rectilinear triangulation $T$ of $E^{3}$ with mesh less than $\eta$ and $i$-skeleton $T_{i}$, and an $\eta$-homeomorphism $h$ of $E^{3}$ onto itself which is the identity outside an $\eta$-neighborhood of $f(D)$ so that $\left(h f(D), h(X), T_{2}, \eta\right)$ has Property $Q$. Furthermore if $G_{\mathrm{IV}}$ denotes the graph which consists of $h f(\mathrm{Bd}(D))$ together with the components of $h f(D) \cap T_{2}$ that intersect $T_{1}$, and if $G_{\mathrm{III}}$ denotes $h^{-1}\left(G_{\mathrm{IV}}\right)$, then there is a finite collection $D_{1}^{\mathrm{I}}, \ldots, D_{m}^{\mathrm{I}}, \ldots$ of subdisks of $f(D)$ which have Property $Z^{\prime}\left(\varepsilon_{1}\right)$ so that $G_{\mathrm{I}}=\bigcup \mathrm{Bd}\left(D_{m}^{\mathrm{I}}\right)$ is a stable subgraph of $G_{\mathrm{III}}$. For each $D_{m}^{\mathrm{I}}$ let $D_{m}^{\mathrm{II}}$ denote the $\varepsilon_{1}+2 \eta<2 \varepsilon_{1}$-disk $h\left(D_{m}^{\mathrm{I}}\right)$, let $G_{\mathrm{II}}$ denote the graph $h\left(G_{\mathrm{I}}\right)$, and let $G_{\mathrm{IIO}}$ denote the connected (Lemma 4.2) subgraph $\mathrm{Cl}\left(G_{\mathrm{II}} \cap h f(\operatorname{Int}(D))\right.$ ).

Let $\gamma$ be a positive number. Consider the graph $f^{-1}\left(G_{I}\right)$. Let $G$ be a polyhedral graph in $D$ which is so close to $f^{-1}\left(G_{\mathrm{I}}\right)$ that there is a $\gamma$-homeomorphism $\theta$ of $D$ onto itself which is the identity on $\mathrm{Bd}(D)$ and which takes $G$ onto $f^{-1}\left(G_{\mathrm{I}}\right)$. Let $G_{O}$ denote the connected graph $\mathrm{Cl}(G \cap \operatorname{Int}(D))$, and for each $D_{m}^{\mathrm{I}}$ let $D_{m}^{0}$ denote the polyhedral disk $\theta^{-1} f^{-1}\left(D_{m}^{I}\right)$. We assume that $\varepsilon_{1}$ and $\gamma$ are sufficiently small so that each $D_{m}^{o}$ has diameter less than $\varepsilon$. Now Condition 1 is satisfied in the conclusion of the theorem.

Step 2. Converting pwl approximations to $f$ into pwl approximations to $h f$. Here we take pwl approximations $f_{0}$ and $f_{1}$ to $f$, convert them to pwl approximations to $h f$, and adjust these new approximations slightly so that they carry $D$ onto polyhedral disks which intersect $T_{2}$ in a nice way.

Let $T^{\prime}, T^{\prime \prime}$, and $T^{m}$ be subdivisions of $T$ as indicated in $\S 6$. For each $m$ let $r_{m}$, $L\left(r_{m}\right)$, and $N_{o}\left(r_{m}, T^{m}\right)$ be defined as in $\S 6$. Let $\Delta_{1}, \ldots, \Delta_{j}, \ldots$ denote the 2-simplexes of $T$, and let $t_{1}, \ldots, t_{i}, \ldots$ denote the arc components of the $\left(G_{\mathrm{IIO}} \cap \Delta_{j}\right)$ 's. Let $p_{1}, \ldots, p_{k}, \ldots$ denote the points of $h f(D) \cap T_{1}$.

Let $\delta_{1}$ be a positive number. Use [4], [19] to find a pwl homeomorphism $h_{a}$ of $E^{3}$ onto itself such that $d\left(h_{a}, h\right)<\delta_{1} / 2$ and $d\left(h_{a}, I\right)<\eta$.

Now let $f_{0}$ and $f_{1}$ be pwl homeomorphisms of $D$ onto disjoint polyhedral disks $D_{0}$ and $D_{1}$ in $E^{3}$ such that $d\left(f, f_{e}\right)<\delta(e=0,1)$. Lemma 6.1 shows that if $\delta$ is 
sufficiently small then $d\left(h_{a} f_{e}, h f\right)<\delta_{1}(e=0,1)$. Let $h_{b}$ be a pwl $\delta_{1}$-homeomorphism of $E^{3}$ onto itself so that $h_{b} h_{a}\left(D_{0} \cup D_{1}\right)$ is in general position with respect to $T_{2}$.

Let $\varepsilon_{2}$ be a positive number such that $\varepsilon_{2}$-neighborhoods of the $p_{k}$ 's are mutually exclusive and miss $T_{0}$. From Lemma 5.2 we can require $\delta_{1}$ to be sufficiently small so that there is a pwl $\varepsilon_{2}$-homeomorphism $h_{c}$ of $E^{3}$ onto itself so that $h_{c} h_{b} h_{a}\left(D_{0} \cup D_{1}\right)$ is in general position with respect to $T_{2}$ and $h_{c} h_{b} h_{a}\left(D_{e}\right) \cap T_{1}(e=0,1)$ consists of points $p_{k e}$ where each $p_{k e}$ is contained in an $\varepsilon_{2}$-neighborhood of $p_{k}$. Let $G_{\mathrm{IV}}^{e}(e=0,1)$ denote the graph which consists of $h_{c} h_{b} h_{a}\left(\mathrm{Bd}\left(D_{e}\right)\right)$ together with the components of $h_{c} h_{b} h_{a}\left(D_{e}\right) \cap T_{2}$ that intersect $T_{1}$.

Let $\varepsilon_{3}$ be a positive number such that $\varepsilon_{3}$-neighborhoods of the components of the $\left(h(X) \cap \Delta_{j}\right)$ 's are mutually exclusive. Now $d\left(h_{c} h_{b} h_{a} f_{e}, h f\right)<\delta_{1}+\delta_{1}+\varepsilon_{2}(e=0,1)$. From Lemma 5.3 we find that by requiring both $\delta_{1}$ and $\varepsilon_{2}$ to be sufficiently small there is a homeomorphism $\pi_{e}^{\prime}(e=0,1)$ of $G_{\mathrm{IV}}$ onto $G_{\mathrm{IV}}^{e}$ which takes each $p_{k}$ onto $p_{k e}$, which takes each component $t$ of a $h(X) \cap \Delta_{f}$ onto an arc in $h_{c} h_{b} h_{a}\left(D_{e}\right) \cap \Delta_{j}$ that lies in an $\varepsilon_{3}$-neighborhood of $t$, and which takes each component $s$ of $h f(\mathrm{Bd}(D))-\mathrm{Cl}\left(G_{\mathrm{IV}} \cap h f(\operatorname{Int}(D))\right)$ onto a component of

$$
h_{c} h_{b} h_{a}\left(\mathrm{Bd}\left(D_{e}\right)\right)-\mathrm{Cl}\left(G_{\mathrm{IV}}^{e} \cap h_{c} h_{b} h_{a}\left(\operatorname{Int}\left(D_{e}\right)\right)\right)
$$

that lies in an $\varepsilon_{3}$-neighborhood of $s$. For each $i$ set $t_{i}^{e}=\pi_{e}^{\prime}\left(t_{i}\right)$ and for each $m$ set $r_{m}^{e}=\pi_{e}^{\prime}\left(r_{m}\right)$.

Let $\varepsilon_{4}$ be a positive number. From Lemma 6.6 we find that $\delta_{1}, \varepsilon_{1}, \varepsilon_{2}$, and $\varepsilon_{3}$ can be required to be sufficiently small so that $\pi_{e}^{\prime} \mid G_{\mathrm{II}}(e=0,1)$ can be extended to an $\varepsilon_{4}$-homeomorphism $\pi_{e}$ of $h f(D)$ onto $h_{c} h_{b} h_{a}\left(D_{e}\right)$ where each $\pi_{e}\left(D_{m}^{\mathrm{II}}\right)$ has diameter less than $\varepsilon_{4}$, where each $r_{m}^{e} \subset L\left(r_{m}\right) \cap \operatorname{Int}\left(N\left(r_{m}, T^{\prime \prime}\right)\right)$, and where each

$$
r_{m}^{e} \cup \operatorname{Int}\left(N_{o}\left(r_{m}, T^{m}\right)\right)
$$

contains a neighborhood of $r_{m}^{e}$ in $\pi_{e}\left(D_{m}^{\mathrm{II}}\right)$.

Step 3. A trial embedding of $G_{O} \times[0,1]$. In this step we adjust $h_{c} h_{b} h_{a}\left(D_{0} \cup D_{1}\right)$ slightly by a homeomorphism $h_{d}$ of $E^{3}$ which keeps $\pi_{0}\left(G_{O}\right) \cup \pi_{1}\left(G_{O}\right)$ fixed, and then we construct a trial embedding $g^{\prime}: G_{O} \times[0,1] \rightarrow E^{3}$ so that $g^{\prime}\left(G_{O} \times(0,1)\right)$ misses $h_{d} h_{c} h_{b} h_{a}\left(D_{0} \cup D_{1}\right)$.

For each $\Delta_{j}$ and each $t_{i}$ in $h f(\operatorname{Int}(D)) \cap \Delta_{j}$ that has both endpoints on $\operatorname{Bd}\left(\Delta_{j}\right)$ let $F_{i}$ denote the polyhedral disk in $N\left(t_{i}, T^{m}\right) \cap \Delta_{j}$ whose boundary consists of $t_{i}{ }^{0} \cup t_{i}{ }^{1}$ together with two arcs in $N\left(t_{i}, T^{m}\right) \cap \operatorname{Bd}\left(\Delta_{j}\right)$. For each $\Delta_{j}$ and each $t_{i}$ in $\Delta_{j}$ that has only one endpoint on $\operatorname{Bd}\left(\Delta_{j}\right)$ let $F_{i}$ be a polyhedral disk in $N\left(t_{i}, T^{m}\right) \cap \Delta_{j}$ whose boundary contains $t_{i}^{0} \cup t_{i}^{1}$, whose intersection with $\mathrm{Bd}\left(\Delta_{j}\right)$ is the arc in $N\left(t_{i}, T^{\prime \prime}\right) \cap \mathrm{Bd}\left(\Delta_{j}\right)$ between $t_{i}^{0} \cap \mathrm{Bd}\left(\Delta_{j}\right)$ and $t_{i}^{1} \cap \mathrm{Bd}\left(\Delta_{j}\right)$, and whose intersection with $h_{c} h_{b} h_{a}\left(D_{0} \cup D_{1}\right)$ is $t_{i}^{0} \cup t_{i}^{1}$. See Figure 7.1. Since each $t_{i}$ underlies a subcomplex of $T^{\prime}$ the $F_{i}$ 's associated with a given $\Delta_{j}$ are mutually exclusive.

We wish to have each disk $F_{i}$ as the image of $t_{i} \times[0,1]$ under the trial embedding $g^{\prime}$. Thus for $g^{\prime}$ to have the properties indicated at the beginning of this step we need to clear each $F_{i}-\left(\pi_{0}\left(t_{i}\right) \cup \pi_{1}\left(t_{i}\right)\right)$ of points of $h_{c} h_{b} h_{a}\left(D_{0} \cup D_{1}\right)$. We do this in the next two paragraphs. 

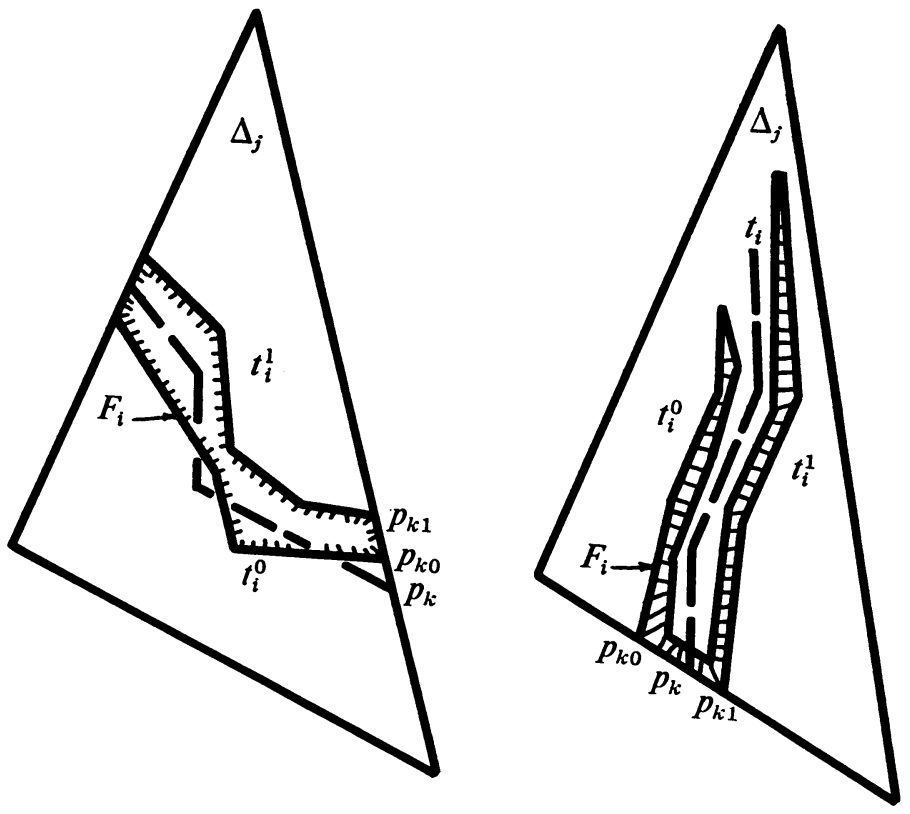

FIGURE 7.1

For each $t_{i}$ which has both endpoints on $T_{1}$ let $F_{i,-1}$ and $F_{i, 1}$ be a pair of polyhedral disks missing $T_{2}$ which lie to either side of $F_{i}$ so that $F_{i,-1} \cup F_{i, 1}$ separates in $h_{c} h_{b} h_{a}\left(D_{e}\right)(e=0,1), \pi_{e}\left(G_{\text {IIO }}\right)$ from every point of $h_{c} h_{b} h_{a}\left(D_{e}\right) \cap \operatorname{Int}\left(F_{i}\right)$. We assume that the $F_{i j}$ 's are mutually exclusive. Fatten up the $F_{i j}$ 's into mutually exclusive polyhedral cubes missing $T_{2}$ whose boundaries $R_{i j}$ are 2-spheres in general position with respect to $h_{c} h_{b} h_{a}\left(D_{0} \cup D_{1}\right)$. Let $U_{e}(e=0,1)$ denote the component of $h_{c} h_{b} h_{a}\left(D_{e}\right)-\bigcup_{i, j} R_{i j}$ which contains the connected graph $\pi_{e}\left(G_{\text {IIO }}\right)$. Since the $F_{i j}$ 's separate in $h_{c} h_{b} h_{a}\left(D_{e}\right)(e=0,1), \pi_{e}\left(G_{\mathrm{IIO}}\right)$ from the Int $\left(F_{i}\right)$ 's no point of $U_{e}$ can lie in an Int $\left(F_{i}\right)$. See Figure 7.2.

Each $R_{i j}$ is contained in a 3-simplex of $T$ and so has diameter less than $\eta<\varepsilon_{4}$. Each component of $h_{c} h_{b} h_{a}\left(D_{e}\right)-U_{e}(e=0,1)$ lies in some $\pi_{e}\left(D_{m}^{\mathrm{I}}\right)$ and so has diameter less than $\varepsilon_{4}$. We apply Lemma 2.9 to find a pwl $13 \varepsilon_{4}$-homeomorphism $h_{d}$ of $E^{3}$ onto itself which is the identity on $\mathrm{Cl}\left(U_{0} \cup U_{1}\right)$ and on all the $F_{i}$ 's that intersect $h_{c} h_{b} h_{a}\left(\mathrm{Bd}\left(D_{0}\right) \cup \mathrm{Bd}\left(D_{1}\right)\right)$ so that each component of $h_{d} h_{c} h_{b} h_{a}\left(D_{e}\right)$ $-\mathrm{Cl}\left(U_{e}\right)(e=0,1)$ lies in some Int $\left(R_{i j}\right)$. Each Int $\left(F_{i}\right)$ is free of points of

$$
h_{d} h_{c} h_{b} h_{a}\left(D_{0} \cup D_{1}\right) \text {. }
$$

Construct a pwl homeomorphism $g^{\prime}$ of $G_{O} \times[0,1]$ into $E^{3}$ as follows. Define $g^{\prime}$ on $G_{O} \times\{0,1\}$ so that for each point $y=(h f \theta)^{-1}\left(p_{k}\right), g^{\prime}(y, e)=p_{k e}=\pi_{e}\left(p_{k}\right)$, and so that $g^{\prime}$ takes each arc $\left((h f \theta)^{-1}\left(t_{i}\right)\right) \times e(e=0,1)$ pwl onto $t_{i}^{e}$. Define $g^{\prime}$ on each $(h f \theta)^{-1}\left(p_{k}\right) \times[0,1]$ so that it takes this arc pwl onto the arc in $N\left(p_{k}, T^{m}\right) \cap T_{1}$ between $p_{k 0}$ and $p_{k 1}$. For each endpoint $q(i)$ of a $t_{i}$ that lies in $\operatorname{Bd}(D)$ define $g^{\prime}$ on 


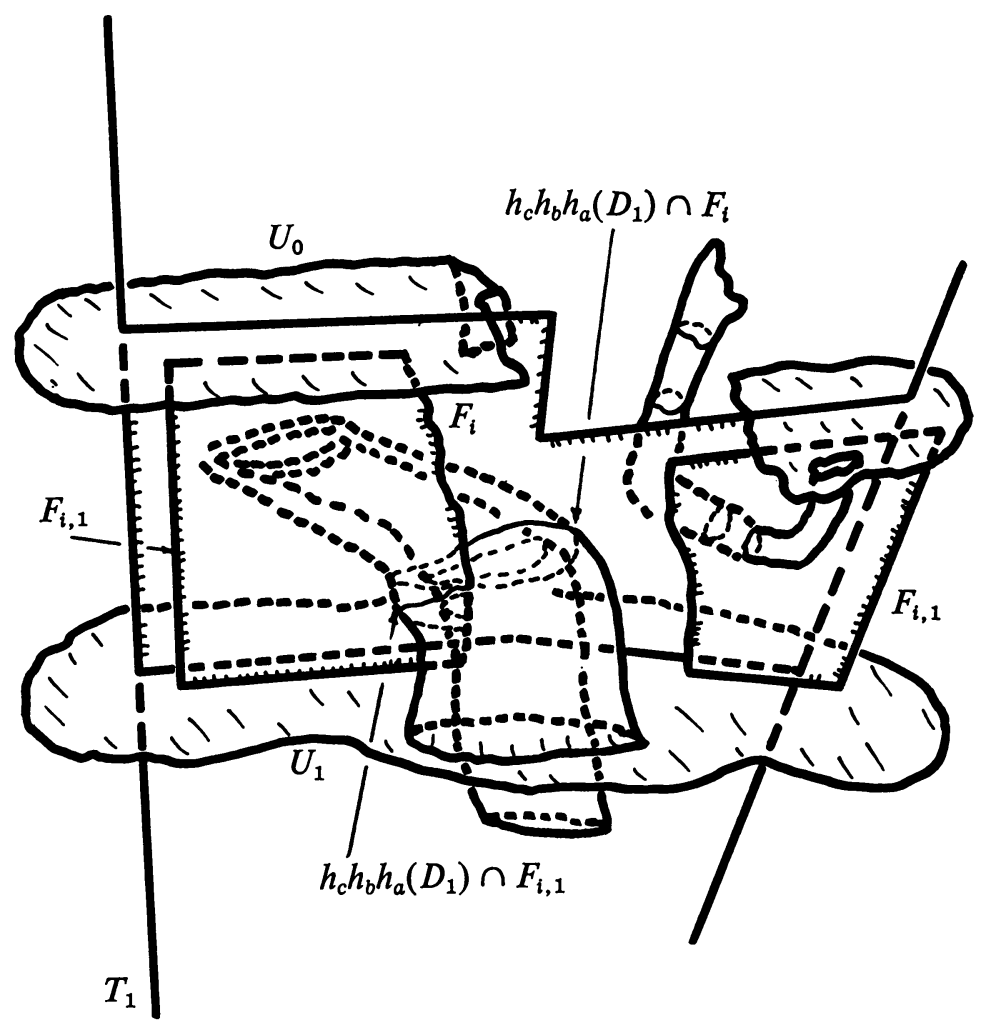

FIGURE 7.2

$\left((h f \theta)^{-1}(q(i))\right) \times[0,1]$ so that it takes this arc pwl onto the part of $\mathrm{Bd}\left(F_{i}\right)-T_{1}$ between $\pi_{0}(q(i))$ and $\pi_{1}(q(i))$. Now $g^{\prime}$ has been defined so that it takes each $\operatorname{Bd}\left(\left((h f \theta)^{-1}\left(t_{i}\right)\right) \times[0,1]\right)$ pwl onto $\mathrm{Bd}\left(F_{i}\right)$. Use Lemma 2.1 to define $g^{\prime}$ so that it takes $\left((h f \theta)^{-1}\left(t_{i}\right)\right) \times[0,1]$ pwl onto $F_{i}$. So defined $g^{\prime}$ takes $G_{O} \times[0,1]$ pwl onto $\cup F_{i}$ and $g^{\prime}\left(G_{O} \times(0,1)\right)$ misses $h_{d} h_{c} h_{b} h_{a}\left(D_{0} \cup D_{1}\right)$.

Step 4. Extending $g^{\prime}$ to a trial embedding of $G \times[0,1]$. For each point $y$ of $G_{O}$, $g^{\prime}(y \times[0,1])$ has diameter less than $\eta$ since $g^{\prime}(y \times[0,1])$ is contained in some 2-simplex of $T$. Furthermore each $h_{d}\left(\pi_{e}\left(D_{m}^{\mathrm{II}}\right)\right)(e=0,1)$ has diameter less than $\varepsilon_{4}+2\left(13 \varepsilon_{4}\right)=27 \varepsilon_{4}$. Thus each set

$$
E_{m}=h_{d}\left(\pi_{0}\left(D_{m}^{\mathrm{I}}\right) \cup \pi_{1}\left(D_{m}^{\mathrm{I}}\right)\right) \cup g^{\prime}\left(\left((h f \theta)^{-1}\left(r_{m}\right)\right) \times[0,1]\right)
$$

has diameter less than $2\left(27 \varepsilon_{4}\right)+\varepsilon_{4}=55 \varepsilon_{4}$. Notice that $E_{m}$ is a disk if $D_{m}^{\mathrm{II}}$ intersects $h f(\mathrm{Bd}(D))$.

From the fact that the $N_{o}\left(r_{m}, T^{\prime \prime}\right)$ 's are mutually exclusive, the fact that each $\pi_{e}\left(r_{m}\right) \cup \operatorname{Int}\left(N_{o}\left(r_{m}, T^{m}\right)\right)(e=0,1)$ contains a neighborhood of $\pi_{e}\left(r_{m}\right)$ in $\pi_{e}\left(D_{m}^{\mathrm{II}}\right)$ (Lemmas 6.5 and 6.6), and the fact that $h_{d}$ is the identity on $\mathrm{Cl}\left(U_{0} \cup U_{1}\right)$ we can use [12, Lemma 2.4] to construct polyhedral cubes $C_{m}$ of diameter less than $55 \varepsilon_{4}$ 
for the $\left(D_{m}^{\mathrm{II}}\right)$ 's that intersect $h f(\mathrm{Bd}(D))$ so that each $\mathrm{Bd}\left(C_{m}\right)$ contains $E_{m}$ and so that the $\left(C_{m}-E_{m}\right)$ 's are mutually exclusive sets which miss

$$
h_{d} h_{c} h_{b} h_{a}\left(D_{0} \cup D_{1}\right) \cup g^{\prime}\left(G_{0} \times[0,1]\right) .
$$

Define $g^{\prime}$ on $\operatorname{Bd}(D) \times\{0,1\}$ so that it extends $g^{\prime} \mid\left(G_{O} \cap \mathrm{Bd}(D)\right) \times\{0,1\}$ and so that it sends each $\left(D_{m}^{0} \cap \operatorname{Bd}(D)\right) \times e(e=0,1)$ pwl onto $h_{d} \pi_{e}\left(D_{m}^{\mathrm{II}} \cap h f(\operatorname{Bd}(D))\right)$. For each $D_{m}^{0}$ that meets $\mathrm{Bd}(D), g^{\prime}$ takes $\operatorname{Bd}\left(\left(D_{m}^{0} \cap \operatorname{Bd}(D)\right) \times[0,1]\right)$ pwl onto $\operatorname{Bd}\left(E_{m}\right)$. Use Lemma 2.1 to define $g^{\prime}$ so that it takes each $\left(D_{m}^{0} \cap \operatorname{Bd}(D)\right) \times[0,1]$ pwl onto $\mathrm{Bd}\left(C_{m}\right)-\operatorname{Int}\left(E_{m}\right)$. From the construction of the $C_{m}$ 's we see that $g^{\prime}$ is now a pwl homeomorphism of $G \times[0,1]$ into $E^{3}$ such that $g^{\prime}(G \times(0,1))$ misses $h_{d} h_{c} h_{b} h_{a}\left(D_{0} \cup D_{1}\right)$ and for each point $y$ of $G$ the diameter of $g^{\prime}(y \times[0,1])$ is less than $55 \varepsilon_{4}$.

Step 5. The embedding $g$. Let $H_{1}$ denote the homeomorphism $h_{d} h_{c} h_{b} h_{a}$. It is an $\eta+\delta_{1}+\varepsilon_{2}+13 \varepsilon_{4}<16 \varepsilon_{4}$-homeomorphism of $E^{3}$ onto itself. Construct a pwl homeomorphism $f_{e}^{\prime}(e=0,1)$ of $D$ onto $H_{1}\left(D_{e}\right)$ by defining $f_{e}^{\prime}$ first on $G$ so that for each point $y$ of $G, f_{e}^{\prime}(y)=g^{\prime}(y, e)$, and then using Lemma 2.1 to extend $f_{e}^{\prime}$ to all of $D$ so that each $f_{e}^{\prime}\left(D_{m}^{0}\right)=h_{d} \pi_{e}\left(D_{m}^{\mathrm{II}}\right)$.

We suppose now that the $\gamma$ in Step 1 is sufficiently small so that each $h f\left(D_{m}^{0}\right) \cup D_{m}^{\text {II }}$ has diameter less than $\varepsilon_{4}$. Since $h_{d} \pi_{e}(e=0,1)$ is a $14 \varepsilon_{4}$-homeomorphism each $h f\left(D_{m}^{0}\right) \cup h_{d} \pi_{e}\left(D_{m}^{\mathrm{II}}\right)$ has diameter less than $29 \varepsilon_{4}$ so $d\left(h f, f_{e}^{\prime}\right)<29 \varepsilon_{4}$. Then since $h$ is an $\varepsilon_{4}$-homeomorphism of $E^{3}$ we see that $d\left(f, f_{e}^{\prime}\right)<30 \varepsilon_{4}(e=0,1)$ and thus

$$
d\left(f, H_{1}^{-1} f_{e}^{\prime}\right)<30 \varepsilon_{4}+16 \varepsilon_{4}=46 \varepsilon_{4} \quad(e=0,1) .
$$

This shows that $d\left(I, f_{e}\left(H_{1}^{-1} f_{e}^{\prime}\right)^{-1}\right)<46 \varepsilon_{4}+\delta(e=0,1)$.

From Lemma 3.1 we may require $\varepsilon_{4}$ and $\delta$ to be so small that there is a pwl $\varepsilon / 2$-homeomorphism $H_{2}^{e}(e=0,1)$ of $E^{3}$ onto itself such that $H_{2}^{e} \mid D_{e}=f_{e}\left(H_{1}^{-1} f_{e}^{\prime}\right)^{-1}$ and $H_{2}^{e}$ is the identity except on a finite polyhedron $K_{e}$ containing $D_{e}$. There is no loss in assuming that $K_{0}$ does not intersect $K_{1}$. Define a pwl $\varepsilon / 2$-homeomorphism $H_{2}$ of $E^{3}$ by setting $H_{2}=H_{2}^{e}$ on $K_{e}(e=0,1)$ and the identity elsewhere.

The promised embedding $g$ is defined to be $H_{2} H_{1}^{-1} g^{\prime}$. If $y$ is a point of $G$ then $g(y, e)=H_{2} H_{1}^{-1} g^{\prime}(y, e)=H_{2} H_{1}^{-1} f_{e}^{\prime}(y)=f_{e}(y) \quad(e=0,1)$. The diameter of each $g(y \times[0,1])$ is less than $55 \varepsilon_{4}+2\left(16 \varepsilon_{4}\right)+\varepsilon / 2$ so by requiring $\varepsilon_{4}$ to be less than $\varepsilon / 174$ we cause Condition 2 to be satisfied in the conclusion of the theorem. That Condition 3 is satisfied follows from the fact that $g^{\prime}(G \times(0,1))$ misses $H_{1}\left(D_{0} \cup D_{1}\right)$.

This completes the proof of the theorem.

THEOREM 7.2. Suppose that $D$ is a polyhedral 2-cell, $M$ is a pwl 3-manifold, $f$ is a homeomorphism of $D$ into Int $(M)$, and $\varepsilon>0$.

There is a $\delta>0$ such that if $f_{0}$ and $f_{1}$ are pwl homeomorphisms of $D$ onto disjoint polyhedral disks $D_{0}$ and $D_{1}$ in $M$ where $d\left(f, f_{e}\right)<\delta(e=0,1)$, then there is a pwl homeomorphism $g$ of $D \times[0,1]$ into Int $(M)$ so that for each point $y$ of $D, g(y, e)$ $=f_{e}(y)(e=0,1)$ and the diameter of $g(y \times[0,1])$ is less than $\varepsilon$. 
Proof. From [18], $f(D)$ is contained in the interior of a polyhedral cube-withhandles $K$. Since $K$ can be pwl embedded in $E^{3}$ under a uniformly continuous homeomorphism we might as well restrict our proof to the case where $M=E^{3}$.

Let $\varepsilon_{1}<\varepsilon / 4$ be a positive number less than one tenth the diameter of $f(D)$. Let $\varepsilon_{2}<\varepsilon_{1}$ be a positive number such that the image under $f$ of each $\varepsilon_{2}$-set in $D$ has diameter less than $\varepsilon_{1}$. Let $\delta$ be subject to the restrictions on $\delta$ in Theorem 7.1 for the substitution $D \rightarrow D, f \rightarrow f$, and $\varepsilon_{2} \rightarrow \varepsilon$.

Let $f_{0}$ and $f_{1}$ be pwl homeomorphisms of $D$ onto disjoint polyhedral disks $D_{0}$ and $D_{1}$ and $E^{3}$ such that $d\left(f, f_{e}\right)<\delta(e=0,1)$. From Theorem 7.1 there is a finite collection $D_{1}^{0}, \ldots, D_{m}^{0}, \ldots$ of polyhedral subdisks of $D$ which has Property $Z\left(\varepsilon_{2}\right)$ so that $G=\bigcup \operatorname{Bd}\left(D_{m}^{0}\right)$ is a stable graph. Further there is a pwl homeomorphism $g^{\prime}$ of $G \times[0,1]$ into $E^{3}$ such that $g^{\prime}(G \times[0,1]) \cap D_{e}=g^{\prime}(G \times e)(e=0,1)$ and for each point $y$ in $G, g^{\prime}(y, e)=f_{e}(y)(e=0,1)$ and the diameter of $g^{\prime}(y \times[0,1])$ is less than $\varepsilon_{2}$. Define $g$ on $D \times\{0,1\}$ so that for each point $y$ of $D, g(y, e)=f_{e}(y)(e=0,1)$. Set $g=g^{\prime}$ on $G \times[0,1]$.

For each disk $D_{m}^{0}$ consider the polyhedral 2-sphere $g\left(\operatorname{Bd}\left(D_{m}^{0} \times[0,1]\right)\right)$. It bounds [15] a pwl 3-cell $C_{m}$. Use Lemma 2.1 to extend $g$ so that it takes $D_{m}^{0} \times[0,1]$ pwl onto $C_{m}$.

No two of the $C_{m}$ 's share interior points for if some Int $\left(C_{i}\right) \cap \operatorname{Int}\left(C_{j}\right)$ were nonempty $(i \neq j)$ there would be a point say of $D_{0}-f_{0}\left(D_{i}^{0}\right)$ in Int $\left(C_{i}\right)$. But since $D_{0}-f_{0}\left(D_{i}^{0}\right)$ is connected $D_{0}-f_{0}\left(D_{i}^{0}\right)$ would be contained in Int $\left(C_{i}\right)$ so $D_{0}$ would be contained in $C_{i}$. This is impossible since the diameter of $D_{0}$ exceeds $10 \varepsilon_{1}-2 \delta>8 \varepsilon_{1}$ and the diameter of $C_{i}$ is less than $\varepsilon_{1}+2 \delta+\varepsilon_{1}<4 \varepsilon_{1}$. Thus $g$ is a pwl homeomorphism of $D \times[0,1]$ into $E^{3}$ such that for each point $y$ of $D, g(y, e)=f_{e}(y)(e=0,1)$. Since each $C_{m}$ has diameter less than $4 \varepsilon_{1}<\varepsilon$ each $g(y \times[0,1])$ has diameter less than $\varepsilon$.

By using two dimensional techniques analogous to those used in the proofs of Theorem 7.1 and Theorem 7.2 we obtain the following two dimensional version of Theorem 7.2.

TheOREM 7.3. Suppose that $A$ is a polygonal arc, $M$ is a pwl 2-manifold, $f$ is a homeomorphism of $A$ into Int $(M)$, and $\varepsilon>0$.

There is $a \delta>0$ such that if $f_{0}$ and $f_{1}$ are pwl homeomorphisms of $A$ onto disjoint polygonal arcs $A_{0}$ and $A_{1}$ in Int $(M)$ where $d\left(f, f_{e}\right)<\delta(e=0,1)$, then there is a pwl homeomorphism $g$ of $A \times[0,1]$ into Int $(M)$ so that for each point $y$ of $D, g(y, e)$ $=f_{e}(y)$ and the diameter of $g(y \times[0,1])$ is less than $\varepsilon$.

8. Piecing together cartesian products of disks with $[0,1]$. In this section we introduce a pair of constructions which enable us to prove our general cartesian product theorem by piecing together cartesian products of disks with $[0,1]$.

For the first construction consider a 2-simplex $\Delta=v \sigma$ where $\sigma$ is a 1-simplex with vertices $v_{a}$ and $v_{b}$. Let $\alpha_{a}$ and $\alpha_{b}$ be a pair of positive numbers less than $1 / 3$, and let 
$\sigma(\alpha)$ denote the line segment in $\Delta$ whose endpoints are $\alpha_{a} v+\left(1-\alpha_{a}\right) v_{a}$ and $\alpha_{b} v+$ $\left(1-\alpha_{b}\right) v_{b}$. Let $\Delta(\alpha)$ denote the 2-simplex $v \sigma(\alpha)$, and let $E(\alpha)$ denote the disk $\mathrm{Cl}(\Delta-\Delta(\alpha))$. For each point $p$ of Int $(\sigma)$ let $r_{p}(\alpha)$ denote the line segment in $\Delta$ with one endpoint at $p$ and the other on Int $(\sigma(\alpha))$ which is perpendicular to $\sigma$ (if such a segment exists). Let $p(\alpha)$ denote the point $r_{p}(\alpha) \cap \sigma(\alpha)$. For each pair of points $q_{1}$ and $q_{2}$ in Int ( $\left.\sigma\right)$ let $\sigma_{q_{1} q_{2}}$ denote the part of $\sigma$ between $q_{1}$ and $q_{2}$, and if the $r_{q_{1}}(\alpha)$ 's are defined let $E_{q_{1} q_{2}}(\alpha)$ denote the closure of the component of $E(\alpha)-$ $\left(r_{q_{1}}(\alpha) \cup r_{q_{2}}(\alpha)\right)$ between $r_{q_{1}}(\alpha)$ and $r_{q_{2}}(\alpha)$. Let $\sigma_{q_{1} q_{2}}(\alpha)$ denote the arc $E_{q_{1} q_{2}}(\alpha) \cap \sigma(\alpha)$ (if $E_{q_{1} q_{2}}(\alpha)$ is defined).

Let $f$ be a homeomorphism of $\Delta$ into a pwl 3-manifold $M$ such that $f(\Delta) \cap \operatorname{Bd}(M)$ $=f(\sigma)$. Let $\varepsilon_{1}$ be a positive number. Suppose that $f_{0}$ and $f_{1}$ are pwl homeomorphisms of $\Delta$ onto disjoint polyhedral disks $D_{0}$ and $D_{1}$ in $M$ such that $f_{e}(\Delta) \cap \operatorname{Bd}(M)=f_{e}(\sigma)$ and $d\left(f, f_{e}\right)<\varepsilon_{1}(e=0,1)$. Suppose further that $g$ is a pwl homeomorphism of $(\Delta(\alpha) \cup \sigma) \times[0,1]$ into $M$ such that $g(\Delta(\alpha) \times[0,1]) \subset$ Int $(M), g(\Delta(\alpha) \times(0,1))$ misses $D_{0} \cup D_{1}, g(\sigma \times[0,1]) \subset \mathrm{Bd}(M)$, and for each point $y$ of $\Delta(\alpha) \cup \sigma, g(y, e)=f_{e}(y)$ $(e=0,1)$ and the diameter of $g(y \times[0,1])$ is less than $\varepsilon_{1}$.

For each point $p$ of $\sigma$ where $r_{p}(\alpha)$ is defined let $J_{p}(\alpha)$ denote the simple closed curve $\left(\bigcup f_{e}\left(r_{p}(\alpha)\right)\right) \cup g\left(\operatorname{Bd}\left(r_{p}(\alpha)\right) \times[0,1]\right)$. Let $B(\alpha)$ denote the disk $\left(\cup f_{e}(E(\alpha))\right)$ $\cup g(\sigma(\alpha) \times[0,1])$ and $A(\alpha)$ the annulus $B(\alpha) \cup g(\sigma \times[0,1])$. For each pair of points $q_{1}$ and $q_{2}$ in $\sigma$ for which $E_{q_{1} q_{2}}(\alpha)$ is defined let $B_{q_{1} q_{2}}(\alpha)$ denote the disk $\left(\bigcup f_{e}\left(E_{q_{1} q_{2}}(\alpha)\right)\right)$ $\cup g\left(\sigma_{q_{1} q_{2}}(\alpha) \times[0,1]\right)$ and $A_{q_{1} q_{2}}(\alpha)$ the annulus $B_{q_{1} q_{2}}(\alpha) \cup g\left(\sigma_{q_{1} q_{2}} \times[0,1]\right)$.

\section{LEMMA 8.1. Let $p$ be a point of Int ( $\sigma)$ and $\varepsilon_{2}>0$.}

There is an $\eta>0$ such that if $\alpha_{a}, \alpha_{b}$, and $\varepsilon_{1}$ are less than $\eta$, then there is a polyhedral disk $F_{p}(\alpha)$ in $M$ whose diameter is less than $\varepsilon_{2}$ so that $\operatorname{Bd}\left(F_{p}(\alpha)\right)=J_{p}(\alpha)$ and $\operatorname{Int}\left(F_{p}(\alpha)\right)$ is contained in Int $(M)$ and misses $B(\alpha) \cup g(\Delta(\alpha) \times[0,1])$.

Proof. Let $y_{1}$ and $y_{2}$ be points of Int $(\sigma)$ such that $p \in \operatorname{Int}\left(\sigma_{y_{1} y_{2}}\right)$, and let $y_{3}$ and $y_{4}$ be points of Int $\left(\sigma_{y_{1} y_{2}}\right)$ such that $p \in \operatorname{Int}\left(\sigma_{y_{3} y_{4}}\right)$. Let $G$ denote the disk $v \sigma_{y_{3} y_{4}}$. Let $C$ be a pwl 3-cell which misses $f(v)$ and contains a neighborhood of $f(\sigma)$ in $M$. Let $\varepsilon_{3}<\varepsilon_{2}$ be a positive number such that (1) $2 \varepsilon_{3}$-neighborhoods of $f\left(y_{1}\right), f\left(y_{2}\right)$ and $f(G)$ are mutually exclusive, (2) a $2 \varepsilon_{3}$-neighborhood of $f(p)$ misses a $2 \varepsilon_{3}$ neighborhood of $f(\mathrm{Cl}(\Delta-G))$, (3) a $2 \varepsilon_{3}$-neighborhood of $f(\sigma)$ is contained in $C$, and (4) a $2 \varepsilon_{3}$-neighborhood of $f(v)$ misses $C$.

First we show how to choose $\eta$ so that an $\varepsilon_{3}$-disk $F_{p}(\alpha)$ can be found whose interior misses $A(\alpha) \cup \mathrm{Bd}(M)$. Let $C_{1}$ be a pwl 3-cell of diameter less than $\varepsilon_{3}$ such that $C_{1} \cap \mathrm{Bd}(M)$ is a polyhedral disk $K_{1}$ whose interior contains $f(p)$. Let $q_{1}$ and $q_{2}$ be points of $\sigma$ such that $p \in \operatorname{Int}\left(\sigma_{q_{1} q_{2}}\right)$ and $f\left(\sigma_{q_{1} q_{2}}\right) \subset \operatorname{Int}\left(K_{1}\right)$. Let $C_{2}$ be a pwl 3-cell in Int $\left(C_{1}\right) \cup \operatorname{Int}\left(K_{1}\right)$ such that $C_{2} \cap \mathrm{Bd}(M)$ is a disk $K_{2}$ where $f(p) \in \operatorname{Int}\left(K_{2}\right)$ and $K_{2} \cap f(\sigma) \subset f\left(\operatorname{Int}\left(\sigma_{q_{1} q_{2}}\right)\right)$. Choose $C_{2}$ so that there is a polyhedral disk $L_{2}$ in Bd $\left(C_{2}\right)$ - Int $\left(K_{2}\right)$ such that $L_{2}$ fails to intersect $f\left(\sigma_{p q_{2}}\right)$ and $L_{2} \cap \mathrm{Bd}\left(K_{2}\right)$ is an arc whose interior contains $f\left(\sigma_{q_{2} p}\right) \cap \mathrm{Bd}\left(K_{2}\right)$. 
Choose $\eta<\varepsilon_{3}$ so that if $\alpha_{a}$ and $\alpha_{b}$ are less than $\eta$ then (1) $r_{p}(\alpha), r_{q_{1}}(\alpha)$, and $r_{q_{2}}(\alpha)$ exist, (2) a $3 \eta$-neighborhood of $f\left(r_{p}(\alpha)\right)$ is contained in $C_{2}$, (3) a $3 \eta$-neighborhood of $f\left(E_{q_{1} q_{2}}(\alpha)\right)$ is contained in $C_{1}$, (4) a $3 \eta$-neighborhood of $f(E(\alpha))$ is contained in $C$, (5) a $3 \eta$-neighborhood of $f\left(\mathrm{Cl}\left(E(\alpha)-E_{q_{1} q_{2}}(\alpha)\right)\right)$ fails to intersect $C_{2}$, (6) a $3 \eta$ neighborhood of $f\left(E_{p q_{1}}(\alpha)\right) \cap\left(\mathrm{Bd}\left(C_{2}\right)-\operatorname{Int}\left(K_{2}\right)\right)$ is contained in $L_{2}$, and (7) a $3 \eta$-neighborhood of $f\left(E_{q_{2} p}(\alpha)\right)$ fails to intersect $L_{2}$.

Suppose now that $\alpha_{a}, \alpha_{b}$, and $\varepsilon_{1}$ are less than $\eta$. From the conditions on $\eta$ we find that $J_{p}(\alpha) \subset \operatorname{Int}\left(C_{2}\right) \cup \operatorname{Int}\left(K_{2}\right), A_{q_{1} q_{2}}(\alpha) \subset \operatorname{Int}\left(C_{1}\right) \cup \operatorname{Int}\left(K_{1}\right), A(\alpha) \cap C_{2}$ $\subset \operatorname{Int}\left(A_{q_{1} q_{2}}(\alpha)\right), A_{p q_{1}}(\alpha) \cap\left(\operatorname{Bd}\left(C_{2}\right)-\operatorname{Int}\left(K_{2}\right)\right) \subset \operatorname{Int}\left(L_{2}\right) \cup \operatorname{Int}\left(L_{2} \cap K_{2}\right)$, and $A_{q_{2} p}(\alpha)$ $\cap L_{2}$ is empty. By shifting $C_{2}$ a small amount if necessary we can suppose that $\mathrm{Bd}\left(C_{2}\right)$ is in general position with respect to $A(\alpha)$.

From the preceding remarks the components of $A(\alpha) \cap L_{2}$ are simple closed curves in Int $\left(A_{p q_{1}}(\alpha)\right)$; and one of these curves, call it $J$, is nontrivial in $A_{p q_{1}}(\alpha)$ since $L_{2}$ separates the boundary components of $A_{p q_{1}}(\alpha)$. Now $J$ bounds a polyhedral

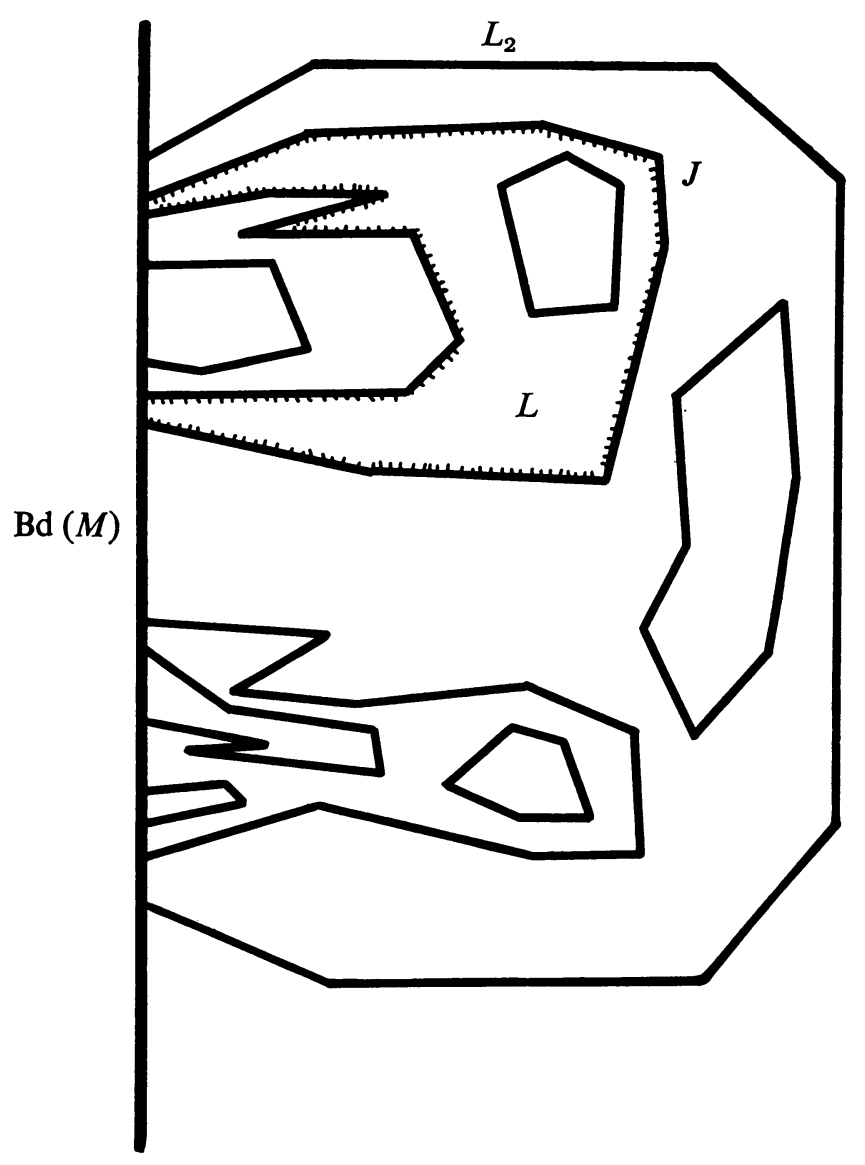

FIGURE 8.1 
disk $L$ in $L_{2}$ and Int $(L) \cap A(\alpha)$ consists of finitely many mutually exclusive simple closed curves in Int $\left(B_{q_{1} q_{2}}(\alpha)\right)$. Thus by repeated applications of [5, Theorem 7.1] as in the proof of [5, Theorem 7.3] we find a pwl homeomorphism $h_{1}$ of $C_{1}$ onto itself which is the identity on $\mathrm{Bd}\left(C_{1}\right)$ and on $J$ so that $h_{1}(\operatorname{Int}(L))$ misses $A(\alpha)$. See Figure 8.1.

To obtain the promised disk we define a pwl homeomorphism $h_{2}$ of $h_{1}(L)$ into $C_{1}$ which slides $J$ onto $J_{p}(\alpha)$ and takes $h_{1}(\operatorname{Int}(L))$ into Int $\left(C_{1}\right)-A(\alpha)$. For $F_{p}(\alpha)$ we take $h_{2} h_{1}(L)$. Since it is contained in $C_{1}$ it has diameter less than $\varepsilon_{3}$.

To insure that Int $\left(F_{p}(\alpha)\right)$ misses $g(\Delta(\alpha) \times[0,1])$ we require $\eta$ to be so small that polyhedral $\varepsilon_{3}$-disks $F_{y_{1}}(\alpha)$ and $F_{y_{2}}(\alpha)$ like $F_{p}(\alpha)$ can also be found. From the conditions on $\eta$ and $\varepsilon_{3}$ the three disks $F_{p}(\alpha), F_{y_{1}}(\alpha)$, and $F_{y_{2}}(\alpha)$ are mutually exclusive, and $A_{y_{1} y_{2}}(\alpha) \cup F_{y_{1}}(\alpha) \cup F_{y_{2}}(\alpha)$ is a polyhedral 2-sphere which bounds a polyhedral cube $C_{3}$ in $C$. Furthermore $F_{p}(\alpha)$ must span $\mathrm{Bd}\left(C_{3}\right)$ from Int $\left(C_{3}\right)$ since otherwise it could not attach onto $J_{p}(\alpha)$ along $g(p \times[0,1])$.

Suppose that there were points of $g(\Delta(\alpha) \times[0,1])$ in Int $\left(F_{p}(\alpha)\right)$. From Condition 2 on $\varepsilon_{3}$ these points would have to belong to the connected set $g((G-\sigma(\alpha)) \times[0,1])$. But by Condition 4 on $\varepsilon_{3}, g(v \times[0,1])$ misses $C_{3}$ so there would have to be points of $g((G-\sigma(\alpha)) \times[0,1])$ in Int $\left(F_{y_{1}}(\alpha)\right) \cup \operatorname{Int}\left(F_{y_{2}}(\alpha)\right)$ which is ruled out by Condition 1 on $\varepsilon_{3}$.

The second construction which we employ is similar to the first one. Consider a pair of 2-simplexes $\Delta_{0}=v_{0} \sigma$ and $\Delta_{1}=v_{1} \sigma$ where $\Delta_{0} \cap \Delta_{1}=\sigma$ and $\sigma$ is a 1-simplex with vertices $v_{a}$ and $v_{b}$. Let $\alpha_{a}$ and $\alpha_{b}$ be a pair of positive numbers less than $1 / 3$. Define $\sigma_{q_{1} q_{2}}$ as before and define $\sigma_{j}(\alpha), \Delta_{j}(\alpha), r_{p j}(\alpha), p_{j}(\alpha), E_{j}(\alpha), E_{q_{1}, q_{2}, j}(\alpha)$, and $\sigma_{q_{1}, q_{2}, j}(\alpha)(j=0,1)$ to correspond to the objects without the subscripts $j$ in the preceding construction.

Let $f$ be a homeomorphism of $\Delta_{0} \cup \Delta_{1}$ into the interior of a pwl 3-manifold $M$. Let $\varepsilon_{1}$ be a positive number. Suppose that $f_{0}$ and $f_{1}$ are pwl homeomorphisms of $\Delta_{0} \cup \Delta_{1}$ onto disjoint polyhedral disks $D_{0}$ and $D_{1}$ in Int $(M)$ such that $d\left(f, f_{e}\right)<\varepsilon_{1}$ $(e=0,1)$. Suppose further that $g$ is a pwl homeomorphism of $\left(\Delta_{0}(\alpha) \cup \Delta_{1}(\alpha)\right) \times[0,1]$ into Int $(M)$ such that $g\left(\left(\Delta_{0}(\alpha) \cup \Delta_{1}(\alpha)\right) \times(0,1)\right)$ misses $D_{0} \cup D_{1}$ and for each point $y$ of $\Delta_{0}(\alpha) \cup \Delta_{1}(\alpha), g(y, e)=f_{e}(y)(e=0,1)$ and the diameter of $g(y \times[0,1])$ is less than $\varepsilon_{1}$.

For each point $p$ of Int $(\sigma)$ for which $r_{p 0}(\alpha)$ and $r_{p 1}(\alpha)$ are defined let $J_{p}(\alpha)$ denote the simple closed curve $\left(\bigcup_{e, j} f_{e}\left(r_{p j}(\alpha)\right)\right) \cup g\left(\left(p_{0}(\alpha) \cup p_{1}(\alpha)\right) \times[0,1]\right)$. Let $A(\alpha)$ denote the annulus $\left(\bigcup_{e, j} f_{e}\left(E_{j}(\alpha)\right)\right) \cup g\left(\left(\sigma_{0}(\alpha) \cup \sigma_{1}(\alpha)\right) \times[0,1]\right)$. For each appropriate pair of points $q_{1}$ and $q_{2}$ of $\sigma$, let $A_{q_{1} q_{2}}(\alpha)$ denote the closed annulus between $J_{q_{1}}(\alpha)$ and $J_{q_{2}}(\alpha)$.

LEMMA 8.2. Let $p$ be a point of Int $(\sigma)$ and $\varepsilon_{2}>0$.

There is a positive number $\eta<\varepsilon_{2}$ such that if $\alpha_{a}, \alpha_{b}$, and $\varepsilon_{1}$ are less than $\eta$, then there is a polyhedral disk $F_{p}(\alpha)$ in $\operatorname{Int}(M)$ of diameter less than $\varepsilon_{2}$ so that $\mathrm{Bd}\left(F_{p}(\alpha)\right)$ $=J_{p}(\alpha)$ and Int $\left(F_{p}(\alpha)\right)$ misses $A(\alpha) \cup g\left(\left(\Delta_{0}(\alpha) \cup \Delta_{1}(\alpha)\right) \times[0,1]\right)$. 
Proof. We only sketch the proof. Details are similar to details of the preceding proof.

Find a small polyhedral 3-cell $C$ whose interior contains $f\left(\sigma_{q p}\right)$ where $q$ is a second point of Int $(\sigma)$ near $p$. Let $x \in \operatorname{Int}\left(\sigma_{q p}\right)$, and let $S$ be a very small polyhedral 2-sphere in Int $(C)$ such that $f(x)$ belongs to the interior of $S$ in $C$ and $f(\sigma) \cap S$ is contained in $f\left(\operatorname{Int}\left(\sigma_{q p}\right)\right)$. By requiring $\eta$ to be very small we find that $S$ separates $J_{x}(\alpha)$ from $J_{p}(\alpha)$ and that $S \cap A(\alpha) \subset \operatorname{Int}\left(A_{q p}(\alpha)\right)$. We can suppose that $S$ has been slightly adjusted so that it is in general position with respect to $A(\alpha)$. Now some component of $S \cap A(\alpha)$ is a simple closed curve $J$ which separates $J_{q}(\alpha)$ from $J_{p}(\alpha)$ in $A(\alpha)$ and bounds a disk $K$ in $S$ such that each component of Int $(K) \cap A(\alpha)$ is a trivial loop in $A_{q p}(\alpha)$. As in the proof of Lemma 8.1 we find a pwl homeomorphism $h$ of $K$ into $C$ so that $h(J)=J_{p}(\alpha)$ and $h(\operatorname{Int}(K))$ fails to intersect $A(\alpha)$. For $F_{p}(\alpha)$ we take $h(K)$.

\section{Building cartesian products of arbitrary surfaces with $[0,1]$.}

THEOREM 9.1. Suppose that $M$ is a pwl 3-manifold, $S$ is a pwl 2-manifold, $R \subset \mathrm{Bd}(S)$ is either a 1-manifold with boundary or the empty set, and $f$ is a homeomorphism of $S$ onto a closed subset of $M$ such that $f(S) \cap \mathrm{Bd}(M)=f(R)$.

Suppose that $\mu$ is a positive continuous function on $S$.

There is a positive continuous function $\nu$ on $S$ such that if $f_{0}$ and $f_{1}$ are pwl homeomorphisms of $S$ onto disjoint polyhedral surfaces $S_{0}$ and $S_{1}$ in $M$ where $f_{e}(S) \cap \operatorname{Bd}(M)$ $=f_{e}(R)(e=0,1)$ and for each point $y$ of $S, \rho\left(f(y), f_{e}(y)\right)<\nu(y)(e=0,1)$, then there is a pwl homeomorphism $g$ of $S \times[0,1]$ into $M$ so that $g(S \times[0,1]) \cap \operatorname{Bd}(M)$ $=g(R \times[0,1])$ and for each point $y$ of $S, g(y, e)=f_{e}(y)(e=0,1)$ and the diameter of $g(y \times[0,1])$ is less than $\mu(y)$.

Proof. It is sufficient to consider the case where $S$ is connected. Further we may assume that for each positive number $t, \mu^{-1}([t, \infty))$ is compact. If $\mu$ does not have this property it can be cut down in size to a continuous function which does have the property.

Consider the two pwl 3-manifolds $M$ and $\mathrm{Bd}(M) \times[0,1]$ where the pwl structure of $\mathrm{Bd}(M)$ is inherited from $M$. By identifying each point $y$ of $\mathrm{Bd}(M)$ with the point $(y, 0)$ of $\mathrm{Bd}(M) \times[0,1]$ we obtain a new pwl 3-manifold $M^{\prime}$ whose interior contains $M$. Give $M^{\prime}$ a metric which extends the metric on $M$. At certain points in this proof we find it convenient to regard $f$ as a homeomorphism of $S$ into $M^{\prime}$.

Let $T$ be a triangulation of $S$ of sufficiently fine mesh so that for each simplex $S$ of $T$ there is a pwl 3-cell $C(s)$ in $M$ whose diameter is less than one third the minimum value of $\mu$ on $N(s, T)$ and which contains a neighborhood of $f(N(s, T))$. We assume that $T$ is such that (1) every component of $R$ contains more than one 1-simplex of $T$, (2) for no vertex $v$ of $T$ is $N(v, T)=S$, and (3) each 2-simplex of $T$ which intersects $\mathrm{Bd}(M)$ intersects it in a 1-simplex or a vertex. From Condition 3 we may assume that $C(s)$ misses $\mathrm{Bd}(M)$ if $s$ misses $R$ and that $C(s)$ intersects 
$\mathrm{Bd}(M)$ in exactly a 2-cell if $s$ intersects $R$. Let $\Delta_{1}, \ldots, \Delta_{i}, \ldots$ denote the 2-simplexes, $\sigma_{1}, \ldots, \sigma_{j}, \ldots$ the 1 -simplexes, and $v_{1}, \ldots, v_{k}, \ldots$ the vertices of $T$. Let $T^{\prime}$ denote the first barycentric subdivision of $T$, and for each simplex $s$ of $T$ let $b(s)$ denote the barycenter of $s$.

For each $\Delta_{i}$ let $H\left(\Delta_{i}\right)$ be a polyhedral 3-manifold with connected boundary such that $H\left(\Delta_{i}\right)$ either misses $\mathrm{Bd}(M)$ or intersects it in a disk, a neighborhood of $H\left(\Delta_{i}\right)$ is contained in $C\left(\Delta_{i}\right)$, and a neighborhood of $f\left(\Delta_{i}\right)$ is contained in $H\left(\Delta_{i}\right)$. Choose the $H\left(\Delta_{i}\right)$ 's so that each $f\left(\mathrm{Cl}\left(S-N\left(\Delta_{i}, T^{\prime}\right)\right)\right)$ misses $H\left(\Delta_{i}\right)$ and $H\left(\Delta_{j}\right)$ misses $H\left(\Delta_{i}\right)$ if $\Delta_{j}$ misses $\Delta_{i}$.

For each $v_{k}$ let $H\left(v_{k}\right)$ be a polyhedral 3-manifold with connected boundary such that $H\left(v_{k}\right)$ either misses $\mathrm{Bd}(M)$ or intersects it in a disk, a neighborhood of $H\left(v_{k}\right)$ is contained in $C\left(v_{k}\right)$, and a neighborhood of $f\left(N\left(v_{k}, T^{\prime}\right)\right)$ is contained in $H\left(v_{k}\right)$. Choose the $H\left(v_{k}\right)$ 's so that each $f\left(\mathrm{Cl}\left(S-N\left(v_{k}, T\right)\right)\right)$ misses $H\left(v_{k}\right)$ and so that $H\left(v_{k}\right)$ misses $H\left(v_{j}\right)$ if $v_{k}$ and $v_{j}$ are not faces of a common simplex of $T$.

For each $\sigma_{j}$ let $H\left(b\left(\sigma_{j}\right)\right)$ be a polyhedral cube such that $H\left(b\left(\sigma_{j}\right)\right)$ either misses $\operatorname{Bd}(M)$ or intersects it in a disk, a neighborhood of $f\left(b\left(\sigma_{j}\right)\right)$ is contained in $H\left(b\left(\sigma_{j}\right)\right)$, and for each $v_{k} \in \sigma_{j}$ a neighborhood of $H\left(b\left(\sigma_{j}\right)\right)$ is contained in $H\left(v_{k}\right)$. Choose the $H\left(b\left(\sigma_{j}\right)\right)$ 's so that they are mutually exclusive and so that $H\left(b\left(\sigma_{j}\right)\right)$ does not intersect $H\left(\Delta_{i}\right)$ or $H\left(v_{k}\right)$ unless $\sigma_{j}$ is a face of $\Delta_{i}$ or $v_{k}$ is a face of $\sigma_{j}$.

From our assumption that $H(s)$ either misses $\mathrm{Bd}(M)$ or intersects it in a disk, that $\mathrm{Bd}(H(s))$ is connected, and that $H(s) \subset C(s)$ it follows that any polyhedral 2-sphere in Int $(H(s)) \cup \operatorname{Int}(H(s) \cap \mathrm{Bd}(M))$ bounds a polyhedral 3-cell in $H(s)$ and hence [15] a pwl 3-cell. Thus any pwl homeomorphism of the boundary of a pwl 3-cell into Int $(H(s)) \cup \operatorname{Int}(H(s) \cap \mathrm{Bd}(M))$ can be extended to a pwl homeomorphism of the 3-cell into $H(s)$ by Lemma 2.1 .

For each $\sigma_{j}$ let $\varepsilon\left(\sigma_{j}\right)$ be a positive number such that a $3 \varepsilon\left(\sigma_{j}\right)$-neighborhood of $f\left(b\left(\sigma_{j}\right)\right)$ is contained in $H\left(b\left(\sigma_{j}\right)\right)$, for every $\Delta_{i}$ containing $\sigma_{j}$ a $3 \varepsilon\left(\sigma_{j}\right)$-neighborhood of $f\left(\Delta_{i}\right)$ is contained in $H\left(\Delta_{i}\right)$, and for every $v_{k} \in \sigma_{j}$ a $3 \varepsilon\left(\sigma_{j}\right)$-neighborhood of $f\left(N\left(v_{k}, T^{\prime}\right)\right)$ is contained in $H\left(v_{k}\right)$. Suppose that $\sigma_{j} \subset R$ and is a face of $\Delta_{i(j)}=v_{k(j)} \sigma_{j}$. In this case let $\eta\left(\sigma_{j}\right)<\frac{1}{3}$ be subject to the restrictions on $\eta$ in Lemma 8.1 when $\Delta_{i(j)}, f \mid \Delta_{i(j)}, M, b\left(\sigma_{j}\right)$, and $\varepsilon\left(\sigma_{j}\right)$ are substituted for the appropriate items. If $\sigma_{j}$ is a face of two 2-simplexes, $\Delta_{i_{0}(j)}=v_{k_{0}(j)} \sigma_{j}$ and $\Delta_{i_{1}(j)}=v_{k_{1}(j)} \sigma_{j}$, let $\eta\left(\sigma_{j}\right)<\frac{1}{3}$ be subject to the restrictions on $\eta$ in Lemma 8.2 when $f \mid \Delta_{i_{0}(j)} \cup \Delta_{i_{1}(j)}, M^{\prime}, b\left(\sigma_{j}\right)$, and $\varepsilon\left(\sigma_{j}\right)$ are substituted for the appropriate items. If $\sigma_{j} \subset \mathrm{Bd}(S)$ but $\sigma_{j} \notin R$ set $\eta\left(\sigma_{j}\right)=1$.

Let $\alpha_{1}, \ldots, \alpha_{k}, \ldots$ be positive numbers such that each $\alpha_{k}<\eta\left(\sigma_{j}\right)$ if $v_{k} \in \sigma_{j}$. For each $\sigma_{j}$ let $v_{k_{a}(j)}$ and $v_{k_{b}(j)}$ denote the endpoints of $\sigma_{j}$. For a 2-simplex $\Delta_{i}=v_{k(j)} \sigma_{j}$ which has $\sigma_{j}$ as a face define a line segment $\sigma_{i j}$ in $\Delta_{i}$ as follows. If $\sigma_{j} \subset \mathrm{Bd}(S)$ but $\sigma_{j} \notin R$ set $\sigma_{i j}=\sigma_{j}$. Otherwise let $\sigma_{i j}$ be the line segment in $\Delta_{i}$ from $\alpha_{k_{a}(j)} v_{k(j)}+$ $\left(1-\alpha_{k_{a}(j)}\right) v_{k_{a}(j)}$ to $\alpha_{k_{b}(j)} v_{k(j)}+\left(1-\alpha_{k_{b}(j)}\right) v_{k_{b}(j)}$. For each $\Delta_{i}$ let $\Delta_{i}(\alpha)$ denote the closure of the component of $\Delta_{i}-\bigcup_{j} \sigma_{i j}$ which contains $b\left(\Delta_{i}\right)$.

For each $\sigma_{j}$ that either lies in $R$ or is not contained in $\mathrm{Bd}(S)$ let $r_{j}$ denote the polyhedral arc such that for every $\Delta_{i}$ that has $\sigma_{j}$ as a face $r_{j} \cap \Delta_{i}$ is the line segment 
in $\Delta_{i}$ from $b\left(\sigma_{j}\right)$ to $\sigma_{i j}$ which is perpendicular to $\sigma_{j}$. For each $v_{k}$ let $D_{k}$ denote the polyhedral 2-cell which is the closure of the component of

$$
N\left(v_{k}, T\right)-\left(\left(\cup \Delta_{i}(\alpha)\right) \cup\left(\cup r_{j}\right)\right)
$$

that contains $v_{k}$. See Figure 9.1. We assume that the $\alpha_{k}$ 's are so small that for each $\sigma_{j}$ and each $v_{k} \in \sigma_{j}$ a $2 \varepsilon\left(\sigma_{j}\right)$-neighborhood of $f\left(D_{k}\right)$ is contained in $H\left(v_{k}\right)$, and for each $r_{f}$ a $2 \varepsilon\left(\sigma_{j}\right)$-neighborhood of $f\left(r_{j}\right)$ is contained in $H\left(b\left(\sigma_{j}\right)\right)$.

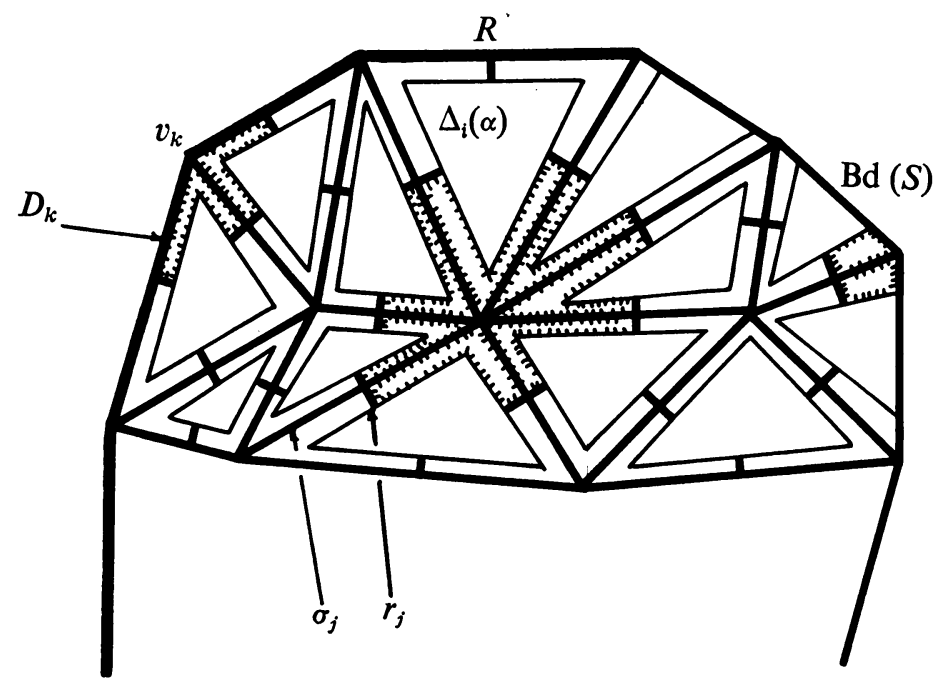

FIGURE 9.1

For each $\Delta_{i}$ let $\varepsilon\left(\Delta_{i}\right)$ be a positive number such that $(1) \varepsilon\left(\Delta_{i}\right)<\eta\left(\sigma_{j}\right)$ for every $\sigma_{j}$ that is a face of $\Delta_{i}$, (2) a $2 \varepsilon\left(\Delta_{i}\right)$-neighborhood of $f\left(\Delta_{i}(\alpha)\right)$ is contained in Int $(M)$ and fails to intersect a $2 \varepsilon\left(\Delta_{j}\right)$-neighborhood of $f\left(\Delta_{j}\right)(j \neq i)$, (3) for every $\sigma_{j}$ that is not a face of $\Delta_{i}$ a $2 \varepsilon\left(\Delta_{i}\right)$-neighborhood of $f\left(\Delta_{i}\right)$ fails to intersect $H\left(b\left(\sigma_{j}\right)\right)$, (4) if $v_{j} \in \Delta_{i}$ and $v_{k} \neq v_{j}$ a $2 \varepsilon\left(\Delta_{i}\right)$-neighborhood of $f\left(v_{j}\right)$ misses $H\left(v_{k}\right)$, (5) if $\Delta_{i}=v_{k(j)} \sigma_{j}$ where $\sigma_{j} \subset R$ a $2 \varepsilon\left(\Delta_{i}\right)$-neighborhood of $f\left(v_{k(j)} \sigma_{i j}\right)$ is contained in Int $(M)$, and (6) if a $\sigma_{j}$ is the face of 2-simplexes $\Delta_{i_{0}(j)}=v_{k_{0}(j)} \sigma_{j}$ and $\Delta_{i_{1}(j)}=v_{k_{1}(j)} \sigma_{j}$ then a $2 \varepsilon\left(\Delta_{i_{0}(j)}\right)$ neighborhood of $f\left(v_{k_{0}(j)} \sigma_{i_{0}(j), j}\right)$ misses a $2 \varepsilon\left(\Delta_{i_{1}(j)}\right)$-neighborhood of $f\left(v_{k_{1}(j)} \sigma_{i_{1}(j), j}\right)$.

For each $\Delta_{i}$ that has a face $\sigma_{j}$ in $R$ let $\delta\left(\Delta_{i}\right)>0$ be subject to the restrictions on $\delta$ in Theorem 7.2 when $\Delta_{i}, M^{\prime}, f \mid \Delta_{i}$, and $\varepsilon\left(\Delta_{i}\right)$ are substituted for the appropriate items, and let $\delta\left(\Delta_{i}\right)$ also be subject to the limitations on $\delta$ in Theorem 7.3 when $\sigma_{j}, \operatorname{Bd}(M), f \mid \sigma_{j}$, and $\varepsilon\left(\Delta_{i}\right)$ are substituted for the appropriate items. For each remaining $\Delta_{i}$ use Theorem 7.2 to find $\delta\left(\Delta_{i}\right)$ by substituting $\Delta_{i}, M^{\prime}, f \mid \Delta_{i}$, and $\varepsilon\left(\Delta_{i}\right)$.

Let $\nu$ be a positive continuous function on $S$ whose maximum value on each $\Delta_{i}$ is less than $\delta\left(\Delta_{i}\right)$.

Suppose now that $f_{0}$ and $f_{1}$ are pwl homeomorphisms of $S$ into $M$ such that $f_{e}(S) \cap \operatorname{Bd}(M)=f_{e}(R)(e=0,1)$ and for each point $y$ of $S, \rho\left(f(y), f_{e}(y)\right)<v(y)$ $(e=0,1)$. 
For each $\Delta_{i}$ there is from Theorem 7.2 a pwl homeomorphism $g_{i}$ of $\Delta_{i} \times[0,1]$ into $M^{\prime}$ such that for every point $y$ of $\Delta_{i}, g_{i}(y, e)=f_{e}(y)(e=0,1)$ and the diameter of $g_{i}(y \times[0,1])$ is less than $\varepsilon\left(\Delta_{i}\right)$. For each $\sigma_{j}$ in $R$ there is from Theorem 7.3 a pwl homeomorphism $g_{j}^{\prime}$ of $\sigma_{j} \times[0,1]$ into $\mathrm{Bd}(M)$ such that for every point $y$ of $\sigma_{j}$, $g_{j}^{\prime}(y, e)=f_{e}(y)(e=0,1)$ and the diameter of $g_{j}^{\prime}(y \times[0,1])$ is less than $\varepsilon\left(\Delta_{i}\right)$ if $\sigma_{j}$ is a face of $\Delta_{i}$. Condition 2 on the $\varepsilon\left(\Delta_{i}\right)$ 's shows that the $g_{i}\left(\Delta_{i}(\alpha) \times[0,1]\right)$ 's are mutually exclusive and are contained in Int $(M)$ and that the $g_{i}\left(\Delta_{i}(\alpha) \times(0,1)\right)$ 's miss $S_{0} \cup S_{1}$. Define $g$ on $S \times\{0,1\}$ so that for each point $y$ of $S, g(y, e)=f_{e}(y)(e=0,1)$. Define $g$ on each $\Delta_{i}(\alpha) \times[0,1]$ to be $g_{i}$. For each $\sigma_{j}$ in $R$ define $g$ on $b\left(\sigma_{j}\right) \times[0,1]$ to be $g_{j}^{\prime}$.

Conditions 1,5 , and 6 on the $\varepsilon\left(\Delta_{i}\right)$ 's enable us to apply Lemmas 8.1 and 8.2 and so find for each $\sigma_{j}$ where an $r_{j}$ is defined a polyhedral disk $F_{j}$ of diameter less than $\varepsilon\left(\sigma_{j}\right)$ such that $\operatorname{Bd}\left(F_{j}\right)=g\left(\operatorname{Bd}\left(r_{j} \times[0,1]\right)\right)$ and $\operatorname{Int}\left(F_{j}\right) \subset \operatorname{Int}(M)$ and misses each $g\left(\Delta_{i} \times\{0,1\} \cup \Delta_{i}(\alpha) \times[0,1]\right)$ where $\Delta_{i}$ has $\sigma_{j}$ as a face. From our assumptions about the sizes of the $\alpha_{k}$ 's a neighborhood of each $F_{j}$ is contained in $H\left(b\left(\sigma_{j}\right)\right)$ so the $F_{j}$ 's are mutually exclusive. From Condition 3 on the $\varepsilon\left(\Delta_{i}\right)$ 's the Int $\left(F_{j}\right)$ 's miss all $g\left(\Delta_{i}(\alpha) \times[0,1]\right)$ 's. Use Lemma 2.1 to define $g$ on each $r_{j} \times[0,1]$ so that $g$ takes it pwl onto $F_{j}$.

From our assumptions about the smallness of the $\alpha_{k}$ 's and from Condition 1 on the $\varepsilon\left(\Delta_{i}\right)$ 's we find that for each $v_{k} \in \operatorname{Int}(R)$,

$$
g\left(\operatorname{Bd}\left(\left(D_{k} \cap R\right) \times[0,1]\right)\right) \subset \operatorname{Int}\left(H\left(v_{k}\right) \cap \operatorname{Bd}(M)\right) .
$$

Use Lemma 2.1 to define $g$ on $\left(D_{k} \cap R\right) \times[0,1]$ so that $g$ takes it pwl into Int $\left(H\left(v_{k}\right) \cap \mathrm{Bd}(M)\right)$. Similarly for each $v_{k} \in \mathrm{Bd}(R)$,

$$
g\left(\left(D_{k} \cap R\right) \times\{0,1\} \cup\left(\operatorname{Bd}\left(D_{k} \cap R\right) \cap \operatorname{Int}(R)\right) \times[0,1]\right)
$$

is contained in Int $\left(H\left(v_{k}\right) \cap \mathrm{Bd}(M)\right)$ and we can define $g$ to take the rest of $\left(D_{k} \cap R\right) \times[0,1]$ pwl into Int $\left(H\left(v_{k}\right) \cap \mathrm{Bd}(M)\right)$ as in Step 3 of the proof of Theorem 7.1 so that $g\left(v_{k} \times(0,1)\right)$ misses $g(R \times\{0,1\})$. Condition 1 among the assumptions about the triangulation $T$ of $S$ insures that $g\left(v_{k} \times[0,1]\right) \cap g\left(v_{j} \times[0,1]\right)$ is empty if $v_{k}$ and $v_{j}$ are distinct points of $\mathrm{Bd}(R)$. Since the $H\left(b\left(\sigma_{j}\right)\right)$ 's are mutually exclusive $g \mid R \times[0,1]$ fails to be a homeomorphism only if some

$$
g\left(\operatorname{Int}\left(\left(D_{k_{1}} \cap R\right) \times[0,1]\right)\right) \cap g\left(\operatorname{Int}\left(\left(D_{k_{2}} \cap R\right) \times[0,1]\right)\right) \quad\left(k_{1} \neq k_{2}\right)
$$

is nonempty. But that implies that $g\left(v_{k_{1}} \times[0,1]\right)$ intersects $g\left(\operatorname{Int}\left(\left(D_{k_{2}} \cap R\right) \times[0,1]\right)\right)$ which is impossible by Condition 4 on the $\varepsilon\left(\Delta_{i}\right)$ 's.

For each $v_{k}$ that does not lie in $\mathrm{Cl}(\mathrm{Bd}(S)-R), g$ takes $\mathrm{Bd}\left(D_{k} \times[0,1]\right) \mathrm{pwl}$ into Int $\left(H\left(v_{k}\right)\right) \cup \operatorname{Int}\left(H\left(v_{k}\right) \cap \mathrm{Bd}(M)\right)$ so by our previous remarks we can extend $g$ to take $D_{k} \times[0,1]$ pwl into $H\left(v_{k}\right)$. Let $K$ denote the polyhedron

$$
R \cup \mathrm{Cl}\left(S-\bigcup\left\{D_{k} \mid v_{k} \in \mathrm{Cl}(\mathrm{Bd}(S)-R)\right\}\right) .
$$

For the same reason that $g \mid R \times[0,1]$ is a homeomorphism, $g \mid K \times[0,1]$ is a homeomorphism, and by construction $g(K \times[0,1]) \cap \operatorname{Bd}(M)=g(R \times[0,1])$. 
By using [12, Lemma 2.4] as in the proof of Lemma 2.8 and as in Step 4 of the proof of Theorem 7.1 we find a collection of mutually exclusive polyhedral 3-cells $B_{k}$ for the $v_{k}$ 's in $\mathrm{Cl}(\mathrm{Bd}(S)-R)$ so that each $B_{k} \subset H\left(v_{k}\right)$, each

$$
B_{k} \cap\left(S_{0} \cup S_{1} \cup g(K \times[0,1])\right)
$$

is contained in $\mathrm{Bd}\left(B_{k}\right)$ and is in fact the disk $g\left(\left(D_{k} \times\{0,1\}\right) \cup\left(\left(D_{k} \cap K\right) \times[0,1]\right)\right)$, and each $B_{k}-B_{k} \cap g(R \times[0,1]) \subset \operatorname{Int}(M)$. For those $v_{k}$ 's we use Lemma 2.1 and [15] to extend $g$ so that it takes each $D_{k} \times[0,1] \mathrm{pwl}$ onto $B_{k}$.

Now $g$ is a homeomorphism of $S \times[0,1]$ into $M$ such that $g(S \times[0,1]) \cap \operatorname{Bd}(M)$ $=g(R \times[0,1])$ and for each point $y$ of $S, g(y, e)=f_{e}(y)(e=0,1)$. Since for each $\Delta_{l}$, $g\left(\Delta_{i} \times[0,1]\right) \subset C\left(\Delta_{i}\right) \cup\left(\bigcup\left\{C\left(v_{k}\right) \mid v_{k} \in \Delta_{i}\right\}\right)$, and since these $C$ 's have diameters less than one third of the minimum value of $\mu$ on $\Delta_{i}$, each $g(y \times[0,1])$ has diameter less than $\mu(y)$. From the assumption on $\mu$ that each $\mu^{-1}([t, \infty))(t>0)$ is compact $g(S \times[0,1])$ is a closed subset of $M$ so $g$ is a pwl homeomorphism by Proposition 2.1. This completes the proof of the theorem.

Here is a topological version of Theorem 9.1.

THEOREM 9.2. Suppose that $M$ is a 3-manifold with boundary, $S$ is a surface in $M$ such that $S \cap \mathrm{Bd}(M)=\mathrm{Bd}(S) \cap \mathrm{Bd}(M)=R$ either a 1-manifold with boundary or the empty set, and $\mu$ is a positive continuous function on $S$.

There is a positive continuous function $v$ on $S$ such that if $f_{0}$ and $f_{1}$ are homeomorphisms of $S$ onto disjoint locally tame surfaces in $M$ such that $f_{e}(S) \cap \operatorname{Bd}(M)$ $=f_{e}(R)(e=0,1)$ and for each point $y$ of $S, \rho\left(y, f_{e}(y)\right)<\nu(y)(e=0,1)$, then there is a homeomorphism $g$ of $S \times[0,1]$ onto a locally tame solid in $M$ so that $g(S \times[0,1])$ $\cap \operatorname{Bd}(M)=g(R \times[0,1])$ and for each point $y$ of $S, g(y, e)=f_{e}(y)(e=0,1)$ and the diameter of $g(y \times[0,1])$ is less than $\mu(y)$.

Proof. Since an open subset of $M$ can always be found which contains $S$ as a closed subset it is sufficient to consider the case where $S$ is a closed connected subset of $M$. From [2], [4], $M$ can be triangulated so we may assume that it is pwl 3-manifold. Similarly since surfaces can be triangulated there is a pwl 2-manifold $\Sigma$ and a homeomorphism $f$ of $\Sigma$ onto $S$. Set $R^{\prime}=f^{-1}(R)$.

Let $\nu^{\prime}$ be a positive continuous function on $\Sigma$ which is subject to the restrictions on $\nu$ in Theorem 9.1 when $M, \Sigma, R^{\prime}, f$ and $(\mu f) / 3$ are substituted for the appropriate items. From the proof of Theorem 9.1 we know that for each positive number $t$, $\left(\nu^{\prime}\right)^{-1}([t, \infty))$ is compact. Define $\nu$ to be $\frac{1}{2} \nu^{\prime} f^{-1}$.

Let $\lambda$ be a nonnegative continuous function on $M$ which is positive on $S$ and which is so small that for each point $y$ of $S, \mu(y) / 6$ is greater than the maximum value of $\lambda$ over all points of $M$ whose distances from $y$ do not exceed $2 / 3 \mu(y)$.

Suppose now that $f_{0}$ and $f_{1}$ are homeomorphisms of $S$ into $M$ such that $f_{e}(S) \cap$ $\operatorname{Bd}(M)=f_{e}(R)(e=0,1)$ and for each point $y$ of $S, \rho\left(y, f_{e}(y)\right)<\nu(y)(e=0,1)$. Let $f_{e}^{\prime}(e=0,1)$ denote the homeomorphism $f_{e} f$. For each point $y$ of $\Sigma$ we have $\rho\left(f(y), f_{e}^{\prime}(y)\right)<\frac{1}{2} \nu^{\prime}(y)$. 
Use [2], [20] to find a homeomorphism $H$ of $M$ onto itself such that $H\left(S_{0} \cup S_{1}\right)$ is locally polyhedral and for each point $y$ of $M, \rho\left(y, H^{-1}(y)\right)<\lambda(y)$. We assume that $H$ moves points so little that for each point $y$ of $\Sigma, \rho\left(f(y), H f_{e}^{\prime}(y)\right)<\nu^{\prime}(y)$ $(e=0,1)$. From [15, §9] we may assume that $H f_{e}^{\prime}(e=0,1)$ takes $\Sigma$ pwl onto $H\left(S_{e}\right)$. From the fact that each $\left(\nu^{\prime}\right)^{-1}([t, \infty))(t>0)$ is compact $H\left(S_{e}\right)(e=0,1)$ is a closed subset of $M$ and thus $H f_{e}^{\prime}$ is a pwl homeomorphism of $\Sigma$ into $M$.

Theorem 9.1 provides a pwl homeomorphism $g^{\prime}$ of $\Sigma \times[0,1]$ into $M$ such that $g^{\prime}(\Sigma \times[0,1]) \cap \operatorname{Bd}(M)=g^{\prime}\left(R^{\prime} \times[0,1]\right)$ and for each point $y$ of $\Sigma, g^{\prime}(y, e)=H f_{e}^{\prime}(y)$ $(e=0,1)$ and the diameter of $g^{\prime}(y \times[0,1])$ is less than $(\mu f(y)) / 3$.

Define $g$ by the rule $g(y, t)=H^{-1} g^{\prime}\left(f^{-1}(y), t\right)$. It is a homeomorphism of $S \times[0,1]$ into $M$ such that $g(S \times[0,1]) \cap \operatorname{Bd}(M)=g(R \times[0,1])$. For each point $y$ of $S$ we have $g(y, e)=H^{-1} g^{\prime}\left(f^{-1}(y), e\right)=H^{-1} H f_{e}^{\prime}\left(f^{-1}(y)\right)=f_{e}(y)(e=0,1)$. The diameter of each $g^{\prime}\left(f^{-1}(y) \times[0,1]\right)$ is less than $\mu(y) / 3$ so since $\nu^{\prime}\left(f^{-1}(y)\right)$ is certainly less than $\mu(y) / 3, g^{\prime}\left(f^{-1}(y) \times[0,1]\right)$ is contained in a $2 / 3 \mu(y)$-neighborhood of $f(y)$. From the conditions on $\lambda, H^{-1}$ moves no point of $g^{\prime}\left(f^{-1}(y) \times[0,1]\right)$ by as much as $\mu(y) / 6$ so $g(y \times[0,1])=H^{-1}\left(g^{\prime}\left(f^{-1}(y) \times[0,1]\right)\right)$ has diameter less than

$$
2 / 3 \mu(y)+2 / 6 \mu(y)=\mu(y) .
$$

\section{REFERENCES}

1. J. W. Alexander, On the deformation of an n-cell, Proc. Nat. Acad. Sci. U.S.A. 10 (1924), 26-28.

2. R. H. Bing, Locally tame sets are tame, Ann. of Math. 59 (1954), 145-158.

3. - Approximating surfaces with polyhedral ones, Ann. of Math. 65 (1957), 456-483.

4. - An alternative proof that 3-manifolds can be triangulated, Ann. of Math. 69 (1959), 37-65.

5. - Conditions under which a surface in $E^{3}$ is tame, Fund. Math. 47 (1959), 105-139.

6. - A surface is tame if its complement is 1-ULC, Trans. Amer. Math. Soc. 101 (1961), 294-305.

7. - Each disk in $E^{3}$ contains a tame arc, Amer. J. Math. 84 (1962), 583-590.

8. - Each disk in $E^{3}$ is pierced by a tame arc, Amer. J. Math. 84 (1962), 591-599.

9. - Approximating surfaces from the side, Ann. of Math. 77 (1962), 145-192.

10. M. Brown, Locally flat embeddings of topological manifolds, Ann. of Math. 75 (1962), 331-341.

11. R. Craggs, Small ambient isotopies of a 3-manifold which transform one embedding of a polyhedron into another, Ph.D. thesis, Univ. of Wisconsin, Madison, 1967.

12. _ Improving the intersection of polyhedra in 3-manifolds, Illinois J. Math. 12 (1968), 567-586.

13. - Small ambient isotopies of a 3-manifold which transform one embedding of a polyhedron into another, Fund. Math. (to appear).

14. ——, Extending homeomorphisms between approximating polyhedra, Duke Math. J. (to appear).

15. W. Graeub, Die semilinearen Abbildungen, S.-B. Heidelberger Akad. Wiss. 4 (1950), 205-272.

16. V. K. A. M. Gugenheim, Piecewise linear isotopy and embeddings of elements and spheres. I, Proc. London Math. Soc. (3) 3 (1953), 29-53. 
17. J. M. Kister, Small isotopies in Euclidean spaces and 3-manifolds, Bull. Amer. Math. Soc. 65 (1959), 371-373.

18. D. R. McMillan, Jr., A criterion for cellularity in a manifold. II, Trans. Amer. Math. Soc. 126 (1967), 217-224.

19. E. E. Moise, Affine structures in 3-manifolds. V. The triangulation theorem and Hauptvermutung, Ann. of Math. 56 (1952), 96-114.

20. - Affine structures in 3-manifolds. VIII. Invariance of the knot types; local tame imbedding, Ann. of Math. 59 (1954), 159-170.

21. C. D. Papakyriakopoulos, Dehn's lemma and the asphericity of knots, Ann. of Math. 66 (1957), 1-26.

22. D. E. Sanderson, Isotopy in 3-manifolds. I. Isotopic deformations of 2-cells and 3-cells, Proc. Amer. Math. Soc. 8 (1957), 912-922.

23. J. Stallings, On the loop theorem, Ann. of Math. 72 (1960), 12-19.

24. J. H. C. Whitehead, Simplicial spaces, nuclei and m-groups, Proc. London Math. Soc. 45 (1939), 243-327.

25. E. C. Zeeman, Seminar on combinatorial topology, Inst. Hautes Études Sci. Publ. Math., 1963 (mimeographed notes).

\section{INSTITUTE FOR ADVANCED STUDY,} Princeton, NeW Jersey 